\title{
Diversity of Internal Sensory Neuron Axon Projection Patterns Is Controlled by the POU-Domain Protein Pdm3 in Drosophila Larvae
}

\author{
(1)Cheng Sam Qian, ${ }^{1}$ Margarita Kaplow, ${ }^{2}$ OJennifer K. Lee, ${ }^{2}$ and ${ }^{\circ}$ Wesley B. Grueber ${ }^{1,2,3}$ \\ ${ }^{1}$ Department of Neuroscience, Columbia University, New York, New York 10027, ${ }^{2}$ Department of Physiology and Cellular Biophysics, Columbia University \\ Medical Center, New York, New York 10032, and ${ }^{3}$ Mortimer B. Zuckerman Mind Brain Behavior Institute, Columbia University, New York, New York 10027
}

Internal sensory neurons innervate body organs and provide information about internal state to the CNS to maintain physiological homeostasis. Despite their conservation across species, the anatomy, circuitry, and development of internal sensory systems are still relatively poorly understood. A largely unstudied population of larval Drosophila sensory neurons, termed tracheal dendrite (td) neurons, innervate internal respiratory organs and may serve as a model for understanding the sensing of internal states. Here, we characterize the peripheral anatomy, central axon projection, and diversity of td sensory neurons. We provide evidence for prominent expression of specific gustatory receptor genes in distinct populations of td neurons, suggesting novel chemosensory functions. We identify two anatomically distinct classes of td neurons. The axons of one class project to the subesophageal zone (SEZ) in the brain, whereas the other terminates in the ventral nerve cord (VNC). We identify expression and a developmental role of the POU-homeodomain transcription factor Pdm3 in regulating the axon extension and terminal targeting of SEZ-projecting td neurons. Remarkably, ectopic Pdm3 expression is alone sufficient to switch VNC-targeting axons to SEZ targets, and to induce the formation of putative synapses in these ectopic target zones. Our data thus define distinct classes of td neurons, and identify a molecular factor that contributes to diversification of axon targeting. These results introduce a tractable model to elucidate molecular and circuit mechanisms underlying sensory processing of internal body status and physiological homeostasis.

Key words: axon patterning; Drosophila; interoceptive; pdm3; subesophageal zone; tracheal dendrite

\section{Significance statement}

How interoceptive sensory circuits develop, including how sensory neurons diversify and target distinct central regions, is still poorly understood, despite the importance of these sensory systems for maintaining physiological homeostasis. Here, we characterize classes of Drosophila internal sensory neurons (td neurons) and uncover diverse axonal projections and expression of chemosensory receptor genes. We categorize td neurons into two classes based on dichotomous axon target regions, and identify the expression and role of the transcription factor Pdm3 in mediating td axon targeting to one of these target regions. Our results provide an entry point into studying internal sensory circuit development and function, and establish $\mathrm{Pdm} 3$ as a regulator of interoceptive axon targeting.

\section{Introduction}

Internal influences on the brain control many aspects of behavior and physiology, including feeding, metabolism, osmoregulation,

\footnotetext{
Received July 25, 2017; revised Dec. 23, 2017; accepted Jan. 18, 2018.

Author contributions: C.S.Q., M.K., and W.B.G. designed research; C.S.Q., M.K., and J.K.L. performed research; C.S.Q., M.K., J.K.L., and W.B.G. contributed unpublished reagents/analytic tools; C.S.Q., M.K., and W.B.G. analyzed data; C.S.Q. and W.B.G. wrote the paper.

This work was supported by a National Science and Engineering Research Council of Canada PGS fellowship to C.S.Q., the Searle Foundation, Columbia University, and National Institutes of Health Grant R01 NS061908 to W.B.G. and Grant R24NS086564 to Tom Clandinin and W.B.G. We thank Dr. John Carlson and Dr. Andrea Tichy for anti-Pdm3 and pdm 3 mutant lines; Dr. Cheng-Ting Chien for anti-Pdm3; Drs. Brian McCabe, Tom Clandinin, Daryl Gohl, Marion Silies, Julie Simpson, Richard Mann, Takashi Suzuki, Gerald Rubin, Aljoscha Nern, Jae Young Kwon, and the Bloomington Stock Center for fly stocks; Richard Blazeski for assistance with electron microscopy; Rebecca Vaadia, Anita Burgos, Naureen Ghani, Rosa Bartoletti, and Taylor Nystrom for help with screening GAL4 lines; Mya Win and Nate Carpenter for assistance in mapping of OK282-Gal4; and members of the W.B.G. laboratory for discussions.
}

mood, and cognition. For example, internally detected nutrient signals regulate feeding behavior (Minokoshi et al., 2004; Coll et al., 2007). Internally detected sex peptide alters postmating reproductive behavior (Yang et al., 2009). Across species, these so-called interoceptive signals are conveyed centrally by internal sensory neurons and, in some cases, by direct sampling of chem-

\section{The authors declare no competing financial interests.}

Correspondence should be addressed to either of the following: Dr. Cheng Sam Qian, Department of Neuroscience, Columbia University, 3227 Broadway, Jerome L. Greene Science Center, MC9891, L9-005, New York, NY 10027 E-mail: sq2141@columbia.edu; or Dr. Wesley B. Grueber, Department of Neuroscience, Columbia University, 3227 Broadway, Jerome L. Greene Science Center, MC9891, L9-007, New York, NY 10027, E-mail:wg2135@columbia.edu.

M. Kaplow's present address: Center for Neural Science, New York University, 4 Washington Place, New York, NY 10003.

DOI:10.1523/JNEUROSCI.2125-17.2018

Copyright $\odot 2018$ the authors $\quad 0270-6474 / 18 / 382081-13 \$ 15.00 / 0$ 
icals in the blood within specialized brain cells (Craig, 2003; Critchley and Harrison, 2013). Relative to the classical senses (vision, hearing, smell, taste, touch), much less is known about the development and function of sensory systems that monitor internal state.

The simpler neuroanatomy of the invertebrate nervous system may provide an entry point for understanding interoceptive circuitry. The Drosophila larval peripheral nervous system is comprised of both surface neurons that detect external stimuli, such as touch and heat, and internal sensory neurons with largely unknown functions. Tracheal dendrite (td) neurons are one class of internal sensory neurons. These cells extend sensory terminals along the trachea (the fly respiratory organ), and axons target as yet uncharacterized regions of the CNS (Bodmer and Jan, 1987; Merritt and Whitington, 1995). Axon projection patterns can inform studies of sensory function because positions are often modality-specific (Pfluger et al., 1988; Murphey et al., 1989). However, lack of knowledge about td axon terminal projections hampers the elucidation of connectivity and function.

Transcription factors control neuronal diversification and endow neurons with subtype-specific morphologies, functions, and connectivity. Studies of Drosophila multidendritic sensory neurons have helped identify factors that control diversification of neuronal morphology (Corty et al., 2009). POU domain transcription factors are regulators of neural development in both vertebrate and invertebrate sensory systems (Erkman et al., 1996; McEvilly et al., 1996; Komiyama et al., 2003; Corty et al., 2016). The Drosophila genome encodes five POU factors, POU domain motif 1 (Pdm1), Pdm2, Pdm3, Drifter, and Acj6. Each of these factors shows specific expression in sensory circuits and corresponding roles in sensory system development. Pdm 1 and Pdm2 function in dendritic diversification in the somatosensory system (Corty et al., 2016) and Drifter and Acj6 in olfactory wiring (Komiyama et al., 2003). The POU domain motif 3 (Pdm3) has been shown to regulate odor receptor expression, axon targeting in olfactory neurons and ellipsoid body ring neurons, and glial development (Tichy et al., 2008; Chen et al., 2012; Bauke et al., 2015). Thus, due to their specific expression patterns and broad roles in nervous system development, POU transcription factors are a good candidate gene family for specifying unique aspects of sensory projections, perhaps including the development of interoceptive circuits.

Here, we characterize the peripheral anatomy, central axon projection, and molecular diversity of Drosophila td sensory neurons. Through single-cell labeling, we constructed a map of td central axon projections, revealing diverse targets. We identify two subtypes of tracheal sensory neurons that project to different regions in the CNS. These axon trajectories are unusually complex among peripheral sensory receptors and project to regions of the brain not previously associated with processing of sensory information from abdominal receptors. Using a candidate gene approach revealed by enhancer trap expression, we show that the development of tracheal sensory axon trajectory is regulated by the POU-homeodomain (HD) transcription factor Pdm3. Together with earlier studies, these data support prominent roles for POU-HD transcription factors in the development of somatosensory circuitry. This work introduces a model for studying molecular, developmental, and circuit mechanisms that underlie the sensing of internal body state.

\section{Materials and Methods}

Drosophila stocks. Animals were reared using standard methods. The following stocks were used and were obtained from Bloomington Drosophila
Stock Center unless otherwise indicated: R31D10-LexA, R22C07-Gal4, R35B01-Gal4, R35B01-LexA, R73B01-Gal4, OK282-Gal4 (generated by Cahir J. O'Kane, unpublished data, gift from Brian McCabe), 260-Gal4 (InSITE GAL4 collection), Gr28a-Gal4, Gr28a-QF2 ${ }^{G 4 H}$ (generated using the HACK method) (Lin and Potter, 2016), Gr28b.c-Gal4, Gr32a-Gal4, Gr33a-Gal4, Gr33a $a^{G A L 4}$, Gr33a-QF (gift from Dr. Jae Young Kwon), Gr89a-Gal4, Ir56aGal4, Ir76b-Gal4, Ir76b-QF, ppk-Gal4, tsh-Gal80 (gift from Julie Simpson, Janelia Research Campus), UAS-mCD8-GFP, 13XLexAop2-mCD8-GFP, 20XUAS-IVS- $m C D 8$-GFP, UAS- $m C D 8$-cherry, QUAS- $m C D 8$-GFP, QUASmtdTomato (gift from Dr. Jae Young Kwon), UAS-MCFO-1, UASpdm3.long (gift from John Carlson, Yale University), pdm $3^{\text {foos28 }}$, Elav-Gal4, P(TRiP.HMJ21205)attP40, UAS-Brp.short.mCherry (Schmid et al., 2008) (provided by Dr. Richard Mann, Columbia University), LexAop-Brp. short.cherry (gift from Takashi Suzuki, Tokyo Institute of Technology). Animals of either sex were analyzed at the third instar larval stage.

Generation of clones. MultiColor FlpOut (MCFO) clones were generated essentially as previously described (Nern et al., 2015). GAL4 lines driving expression in td neurons were crossed to UAS-MCFO-1. Early larval progeny were heat-shocked at $38^{\circ} \mathrm{C}$ for $10-15$ min to sparsely label td neurons.

Immunohistochemistry. Immunohistochemistry was performed as previously described (Matthews et al., 2007). Primary antibodies used were chicken anti-GFP (1:1000; Abcam), rabbit anti-DsRed (1:200; Clontech), mouse anti-FasII (1:10, Developmental Studies Hybridoma Bank), mouse anti-Coracle (1:10, Developmental Studies Hybridoma Bank), mouse anti-HA (1:1200, Cell Signaling Technology), rat antiFLAG (1:100, Novus), mouse anti-Elav (1:10, Developmental Studies Hybridoma Bank), rat anti-pdm3 (1:100; gift from John Carlson; RRID: AB_2569865), guinea pig anti-pdm3 (1:100; gift from Cheng-Ting Chien; RRID:AB_2567243), and rabbit anti-V5 epitope, DyLight 549conjugated (1:200, Rockland).

Electron microscopy. To identify td neurons for TEM, $p d m 3^{\mathrm{OK282}}$-Gal4, UAS- $m$ CD8::GFP third instar larvae were dissected in PBS and fixed in $3 \%$ glutaraldehyde. Larvae were then stained with chicken anti-GFP (1:1000, Abcam) primary antibody followed by goat anti-mouse HRP secondary antibody at a 1:200 dilution. For chromogenic detection, larvae were incubated in 1:20 diaminobenzidine, with $0.3 \% \mathrm{NiCl}_{2}$ and $3 \%$ hydrogen peroxide solution. Larvae were postfixed with $1 \%$ osmium tetroxide in $0.1 \mathrm{M}$ phosphate buffer and processed for TEM as previously described (Kim et al., 2012). Similar to the procedure published by Kim et al. (2012), td neurons were identified after larvae were mounted on epon between two plastic slides. Blocks were trimmed according to the area of interest, and longitudinal serial sections were made before viewing grids on a JEOL electron microscope (Kim et al., 2012).

Experimental design and statistical analysis. Axon lengths and ventral nerve cord (VNC) lengths were measured in Fiji and statistically analyzed using the Kruskal-Wallis test, followed by Bonferroni post hoc pairwise comparisons. Axon midline contact frequencies were analyzed using the $\chi^{2}$ tests with Bonferroni correction. Dendritic arbors were traced in Neurolucida (Microbrightfield) and analyzed in Neurolucida Explorer. Dendrite lengths and node counts were statistically analyzed with MannWhitney $U$ test with Bonferroni correction. Statistical analyses were conducted using IBM SPSS Statistics version 20 (for sample sizes, $p$ values, and associated test statistics, see figure legends).

\section{Results}

\section{td neuron sensory dendrites project along specific} tracheal branches

There are two adjacent td neurons in each abdominal hemisegment from A1-A6, named v'td 1 and v'td 2 for their location in the ventral' cluster of sensory neurons, and one td neuron in A7 (Fig. $1 A, B)$. Fitting with the naming of individual sensory neurons according to their ventral to dorsal position, v'td1 is the more ventral of the two neurons. We screened GAL4 and LexA lines from the InSITE (Gohl et al., 2011), Janelia (Jenett et al., 2012), and OK (courtesy of Dr. Brian McCabe, unpublished data) collections for drivers for peripheral sensory neurons, and identified several lines that labeled td neurons (Table 1). GAL4 and LexA 
A
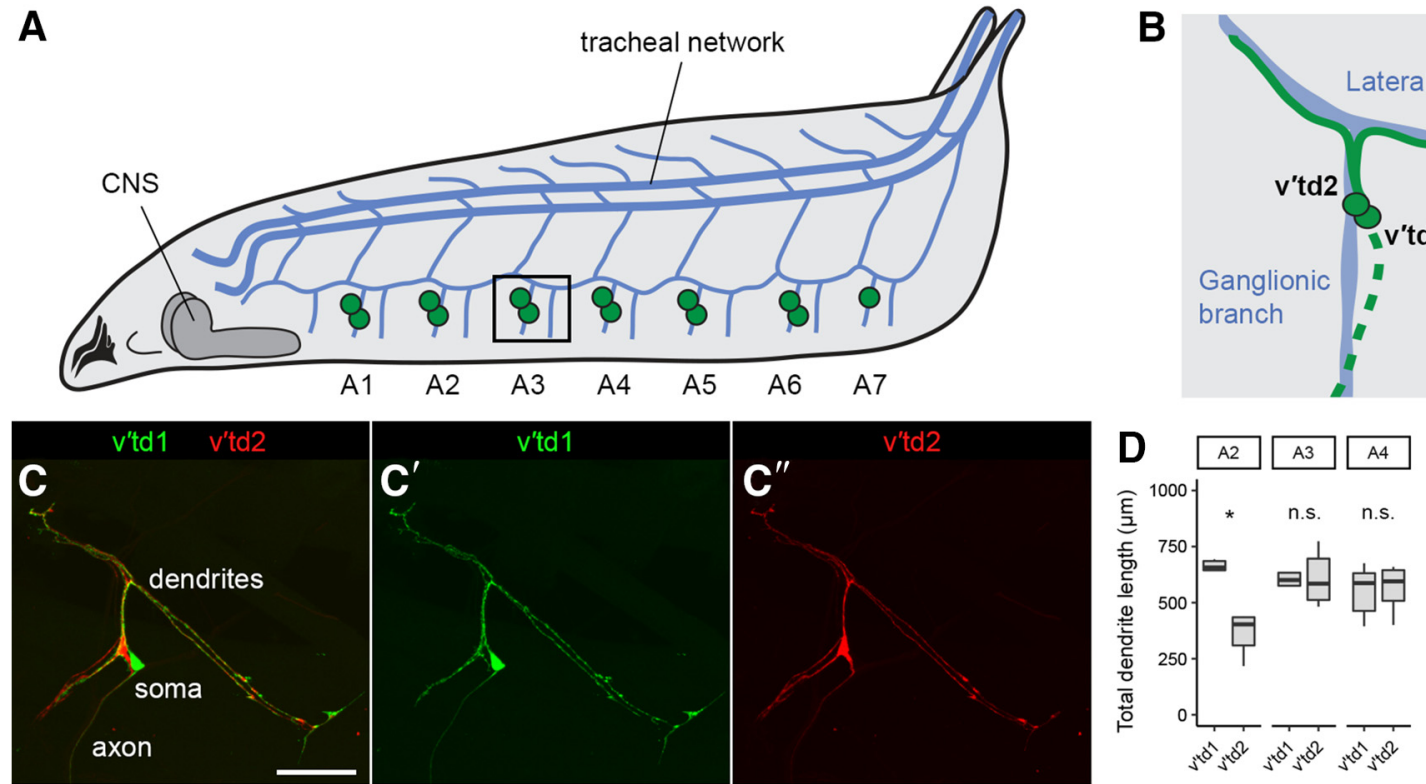

B
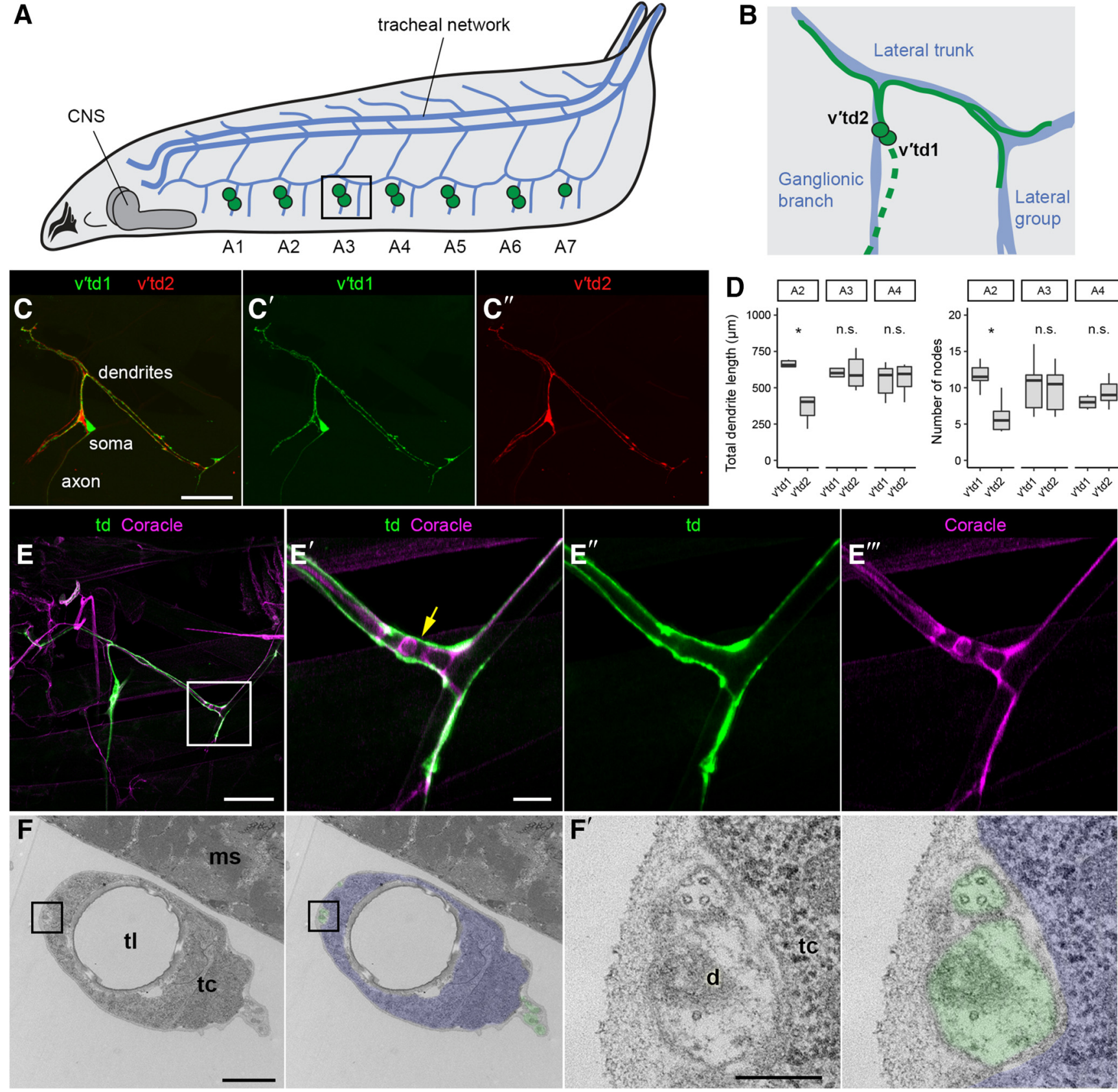

Figure 1. td sensory dendrites associate with tracheal branches. $A$, Schematic of larva showing segmentally repeated td cell bodies (green) relative to the tracheal network. Blue represents only major tracheal branches. $\boldsymbol{B}$, Schematic of $v^{\prime} t d 1$ and v'td2 neurons in one hemisegment, showing cell bodies (green circles), dendrites (solid green lines), and axons (dashed green line), and association with specific tracheal branches (blue). C, Dual-color labeling of v'td1 ( $\boldsymbol{C}^{\prime}$; labeled by R35B01-LexA $>m$ CD8::GFP) and v'td2 ( $\boldsymbol{C}^{\prime}$; labeled by 260-Gal4 $>m$ Cherry) neurons in segment A3. D, Dendrite lengths and node count quantifications for v'td1 (labeled by R35B01-LexA) and v'td2 (labeled by 260-Gal4) neurons in segments A2-A4. Dendrite length was reduced in v'td2 relative to $v^{\prime}$ td1 in segment $A 2$ (Bonferroni adjusted ${ }^{*} p=0.012$, Mann-Whitney $U$ test, $U<0.0001$ ), but not in segments $A 3$ (Bonferroni adjusted $p=1, U=17$ ) or $A 4$ (Bonferroni adjusted $p=1, U=16$ ). Similarly, dendrite nodes were reduced in v'td2 relative to $v^{\prime}$ td 1 in segment $A 2$ (Bonferroni adjusted ${ }^{*} p=0.018$, Mann-Whitney $U$ test, $U=1$ ), but not in segments $A 3$ (Bonferroni adjusted $p=$ $1, U=16.5$ ) or A4 (Bonferroni adjusted $p=0.558, U=10$ ). $n=6$ animals. $E$, td dendrites project along tracheal branches. Coracle labeling shows septate junctions of tracheal epithelial cells. $\boldsymbol{E}^{\prime}-\boldsymbol{E}^{\prime \prime \prime}$, Enlarged image of area boxed in $\boldsymbol{E}$ showing enlargements of td dendrites near tracheal fusion cell (yellow arrow). $\boldsymbol{F}$, Electron micrographs of tracheal tube cross section showing tracheal cell (tc; blue), putative td dendrites (d; green), tracheal lumen (tl), and muscle (ms). $\boldsymbol{F}^{\prime}$, Higher-magnification image of boxed area in $\boldsymbol{F}$, showing two neuronal processes closely associated with the tracheal cell, enclosed by basement membrane. Boxplots represent median (middle line) and 25th to 75th percentile, with whiskers extending to the most extreme data point within 1.5 times the interquartile range of the hinge. Scale bars: $\boldsymbol{C}-\boldsymbol{C}^{\prime \prime}, \boldsymbol{E}, 50 \mu \mathrm{m} ; \boldsymbol{E}^{\prime}-\boldsymbol{E}^{\prime \prime \prime}, 10 \mu \mathrm{m} ; \boldsymbol{F}, 2 \mu \mathrm{m} ; \boldsymbol{F}^{\prime}, 250 \mathrm{~nm}$.

lines typically showed expression in characteristic subsets of $\mathrm{td}$ neurons, suggesting that $t d$ neurons may have diverse identities and functions.

To characterize v'td1 and v'td2 dendrite morphologies, we used GAL4/LexA dual-color labeling. We resolved peripheral arbors with single-cell resolution and characterized their relation- ship to each other and to tracheal branches (Fig. 1C-E). We used antibody against Coracle, a septate junction resident protein, to visualize tracheal cell structure. td cell bodies reside next to the ganglionic branch of the larval tracheal system (nomenclature following Manning and Krasnow, 1993) and extend dendrites along the ganglionic branch and dorsally (Fig. 1C). Major den- 
Table 1. GAL4/LexA driver expression patterns in $\mathrm{td}$ sensory neurons

\begin{tabular}{|c|c|c|c|c|c|c|c|c|c|c|c|c|c|}
\hline & \multicolumn{6}{|c|}{ v'td1 neurons } & \multicolumn{7}{|c|}{ v'td2 neurons } \\
\hline & $\overline{\mathrm{A} 1^{a}}$ & $\mathrm{~A} 2^{a}$ & $\mathrm{~A} 3^{a}$ & $\mathrm{~A} 4^{a}$ & $\mathrm{~A} 5^{a}$ & $\overline{A \sigma^{a}}$ & $\overline{\mathrm{A} 1^{a}}$ & $\mathrm{~A} 2^{a}$ & $\mathrm{~A} 3^{a}$ & $A 4^{b}$ & $A 5^{b}$ & $A 6^{b}$ & $\overline{A 7^{b}}$ \\
\hline OK282-Gal4 & & + & + & + & + & + & + & + & & & & & \\
\hline Gr28a-Gal4 & + & & & & & & + & + & + & & & & \\
\hline Gr28b.c-Gal4 & & & & & & & + & + & + & + & + & + & + \\
\hline Gr33a-Gal4c & & & & & & & & & & + & + & + & \\
\hline Gr89a-Gal4 & & & & & & & + & + & + & + & + & + & \\
\hline |r56a-Gal4 & & & & & + & + & & & & & & & + \\
\hline |r76b-Gal4 & & + & + & + & + & + & & & & & & & \\
\hline R22C07-Gal4 & & & & & & & & & & + & + & + & \\
\hline R31D10-LexA & & & & + & + & + & & & & & & & \\
\hline R35B01-Gal4 & + & + & + & + & + & & + & & & & & & \\
\hline R35B01-LexA & & + & + & + & & & & & & & & & \\
\hline R73B01-Gal4 & & & & & & & + & + & + & + & + & + & + \\
\hline 260-Gal4 & & & & & & & & + & + & + & + & + & + \\
\hline
\end{tabular}

aSEZ targeting td neurons.

${ }^{b}$ VNC targeting td neurons.

${ }^{\mathrm{G}} \mathrm{Gr} 33 a^{\mathrm{Gal}}$ knock-in line shows expression in the same td neurons.

drites bifurcate and extend along the lateral trunk (LT). Branching along the LT tubules was sparse; however, there were local areas of branching and dendrite enlargements, most consistently at tracheal fusion cells (Fig. $1 E$, arrow), which are located along LT where tracheal tubes fuse during development. Dendrites terminate either on the LT or lateral group branch, sometimes with enlargements near or at the termination (Fig. $1 E^{\prime \prime}$ ). We did not observe differences between the trajectories of v'td 1 and v'td 2 in abdominal segments A3 and A4, although v'td2 was relatively simplified in segment A2 (Fig. 1D). v'td1 and v'td 2 dendrites were often fasciculated along the lateral tracheal branches. Dendrites were also tightly associated with tracheal branches along most of their length (Fig. 1E). Using electron microscopy, we identified fasciculated processes on the basal surface of tracheal cells, within a thin basement membrane (Fig. $1 F$ ). Thus, td neurons have mostly similar dendritic morphologies, although GAL4 expression patterns suggested distinct classes of td neurons. We therefore examined td neuron diversity as manifest in axon projection patterns.

\section{td neuron axons terminate in distinct regions of the $\mathrm{VNC}$ and the subesophageal zone (SEZ)}

Sensory neuron diversity is reflected in axon projection patterns (Merritt and Whitington, 1995; Vosshall et al., 2000; Grueber et al., 2007; Kwon et al., 2014), so we examined td axons in greater detail using the collection of GAL4 and LexA lines. Although functions for td neurons are not known, they have been proposed to function as sensors of tracheal status or as proprioceptors based on the location of their dendrites (Bodmer and Jan, 1987). In the fly CNS, the terminal location of axon projections correlates with sensory modality. Within the VNC, nociceptive and touch sensing axons project to ventromedial regions of the neuropil, proprioceptors to more dorsal neuropil, and vibration sensing chordotonal axons to intermediate neuropil (Merritt and Whitington, 1995; Grueber et al., 2007; Zlatic et al., 2009). These somatosensory axons also project primarily within the same VNC neuromere after they enter the CNS (Fig. 2A) (Merritt and Whitington, 1995). By contrast, in embryonic stages, td axons from each segment project together along a far-lateral VNC tract, shift to a medial tract when they reach the thoracic neuromere, and extend further anteriorly (Merritt and Whitington, 1995), although the final targets and mature td axon patterns in the third instar larva have not been described. We labeled a subset of $\mathrm{td}$ neurons using R31D10-LexA > 13XLexAop2-mCD8-GFP, and visualized their axon projections in the third instar CNS with major Fasciclin 2 (Fas2)-positive axonal tracts as landmarks (Landgraf et al., 2003; Grueber et al., 2007). After entering the VNC, td axons project anteriorly along the ventrolateral tract to the T3 neuromere (Fig. $2 A^{\prime}, B$ ). At T3, axons turned medially and dorsally to the dorsomedial fascicle. Thus, td axons make a series of shifts along both the mediolateral and dorsoventral axes as they project to anterior parts of the CNS. Based on these patterns, td axon projections are different from all other characterized types of sensory neurons that innervate the body of the larva (Fig. $\left.2 A, A^{\prime}\right)$.

Prior studies on embryos showed that some td axons extended anteriorly beyond the T3 medial turn (Merritt and Whitington, 1995), but axon terminal locations were not known. To determine their ultimate targets, we examined td axon projections using GAL4 and LexA drivers that labeled subsets of td neurons. The subset of td neurons labeled by R31D10-LexA extended axons beyond the VNC to target the SEZ in the brain (Fig. $2 B, C$ ). By contrast, a different subset of td neurons labeled by R22C07-Gal4 extended axons only to the T3 neuromere in the VNC (Fig. 2 $C^{\prime}$ ). Colabeling of both populations revealed that despite their different targets, VNC and SEZ targeting td axons project along the same initial path in the VNC (Fig. $2 C^{\prime \prime}$ ). Thus, td sensory neurons can be segregated into at least two classes based on axon projections that remain entirely within the $\mathrm{VNC}$ or project anteriorly to the SEZ.

We asked whether SEZ and VNC td axons have different patterns of association or fasciculation as they project through the VNC to their targets, which could help to explain distinct targeting. Notably, although SEZ-targeting and VNC-targeting td axons fasciculate as they enter the VNC from the periphery, once they arrive in the ventrolateral tract axons segregate according to their final targets, such that SEZ-targeting td axons group together and VNC-targeting td axons similarly group together (Fig. $2 D$ ). These data suggest that td axons show hierarchical axon-axon associations as they target to the SEZ and VNC. Initial associations between axons from the same segment may be suppressed as axons encounter other "like-type" axons, which segregates cohorts into distinct subtype-specific bundles en route to their terminal targets.

Given the projection of $t d$ axons to the SEZ, we asked how their terminal targeting relates to gustatory receptor (GR) neuron axons, which strongly innervate the SEZ. Colabeling of td neurons (with R31D10-LexA) and gustatory sensory neurons (with Gr32a-Gal4) showed that SEZ-targeting td axons terminate in a similar location as gustatory sensory axons (Fig. 2E). Compartmentalization of the SEZ is still poorly understood so we cannot eliminate modality-specific fine scale axon organization; nevertheless, the terminal location of these projections raise the possibility of chemosensory roles for td neurons.

td neurons could conceivably make synaptic contacts with targets in an en passant fashion along their entire length, or only in axon terminal regions. To determine whether SEZ and VNC td neurons might have entirely distinct or potentially similar synaptic targets, we expressed the active zone marker Brp.short in different td neuron classes. VNC td neurons labeled by R22C07-Gal4 showed presynaptic zones in VNC neuropil along the ventrolateral tract and near the dorsomedial fascicle (Fig. $2 F$ ). Notably, SEZ td neurons labeled by R31D10-LexA showed Brp.short labeling at the axon terminals in the SEZ, and also along the length of the axons in the VNC (Fig. 2G). These data are consistent with presence of en passant synapses along the length of $\mathrm{td}$ axons. 


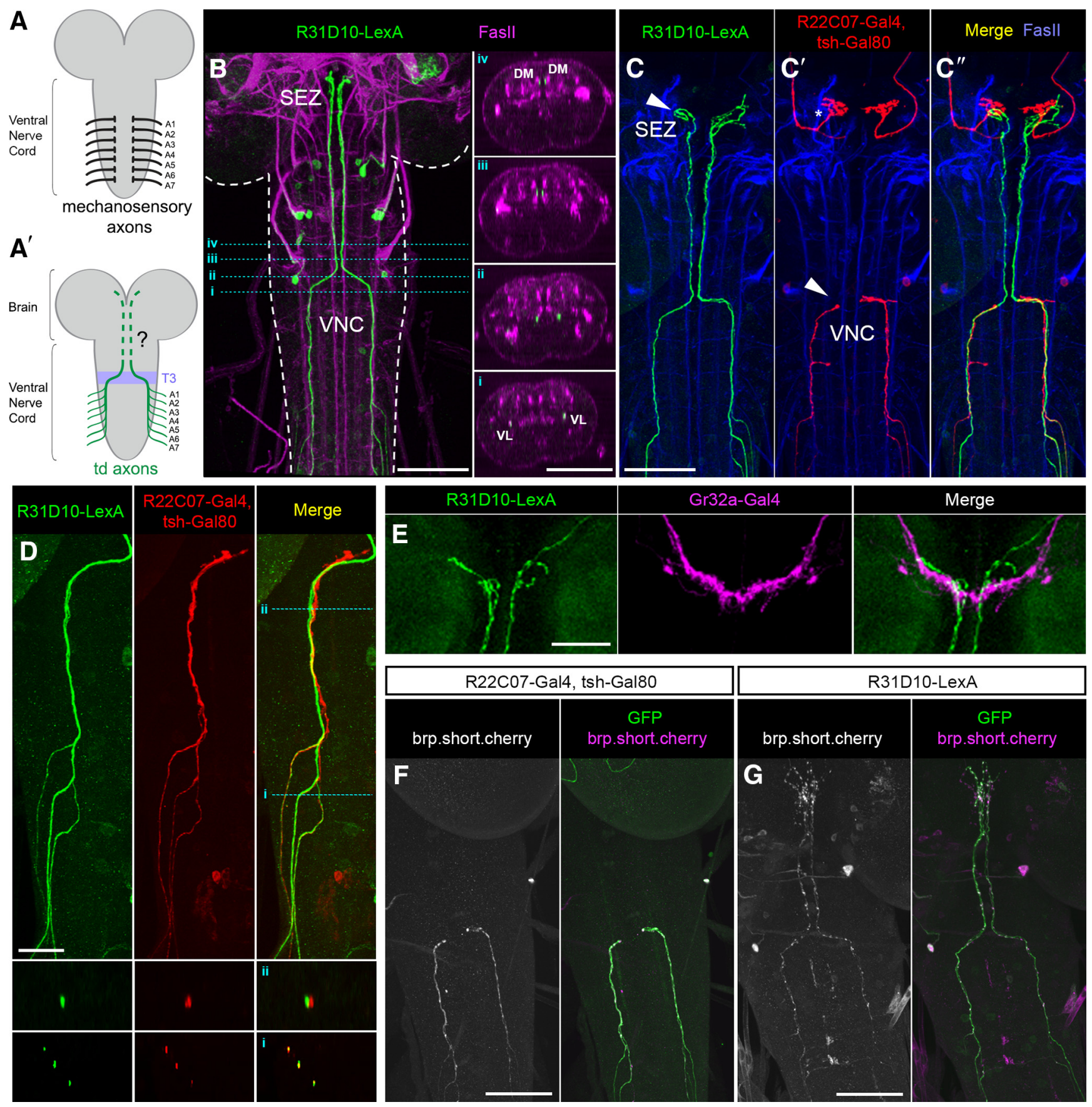

Figure 2. td axons project to the VNC and the SEZ. $\boldsymbol{A}$, Schematic of CNS showing axon trajectories of typical abdominal mechanosensory neurons $(\boldsymbol{A})$, and td neurons $\left(\boldsymbol{A}^{\prime}\right)$. $\boldsymbol{A}^{\prime}$, Dashed line and question mark indicates unresolved terminal trajectory of td axons. Purple band indicates T3 neuromere. $\boldsymbol{B}, \mathrm{td}$ axons labeled by R31D10-LexA $>$ GFP fasciculate and project anteriorly in the CNS (outlined in white). Roman numerals and dotted cyan lines indicate position of cross sections on the right, showing that td axons initially travel anteriorly along the ventrolateral fascicle (Bi), then shift to the dorsomedial fascicle (Biv). C, Two subtypes of td axon projections to the VNC and the SEZ. A subset of td neurons labeled by R31D10-LexA $>m$ CD8::GFP targets the SEZ (C). Another subset of td neurons labeled by R22C07-Gal4 $>m$ Cherry targets the VNC ( C'). Tsh-Gal80 was used to suppress VNC expression in the R22C07-Gal4 driver. R22C07-Gal4 also labels other sensory neurons from the head that project axons to the SEZ (asterisk). D, VNC- and SEZ-targeting axons from the same segment initially fasciculate with each other as they enter the VNC (Di) but segregate into separate bundles in the ventrolateral tract (Dii). Roman numerals and dotted cyan lines indicate position of cross sections on bottom. $\boldsymbol{E}$, SEZ-targeting td axons (green) terminate in close proximity to axon terminals of gustatory sensory neurons (magenta). $\boldsymbol{F}, \boldsymbol{G}$, Presynaptic sites of VNC-targeting td axons $(\boldsymbol{F})$ and SEZ-targeting td axons $(\boldsymbol{G})$ labeled by brp.short.cherry reveal en passant synapses along the td axon bundle tract (labeled by GFP in green). Scale bars: $B, C-C^{\prime \prime}, F, G, 50 \mu \mathrm{m} ; \boldsymbol{D}, \boldsymbol{E}, 20 \mu \mathrm{m}$.

Based on these axon projections and putative presynaptic sites, we identify at least two distinct projection patterns, patterns of axon-axon association, and output sites, for td neurons to the VNC and SEZ. td neuron circuitry appears to be distinct from other known classes of mechanoreceptors, proprioceptors, and nociceptors but may share neuropil targets with other chemosensory neurons. td neurons express GAL4 drivers for chemosensory receptors Given that td axons project to chemosensory recipient regions of the SEZ, we examined whether td neurons express chemosensory receptor genes. A large battery of GR genes have been identified and the expression patterns of many of them can be assessed using available GAL4 lines. A previous analysis of the expression patterns of 68 GRs using Gr-Gal4 drivers identified 7 GRs that 


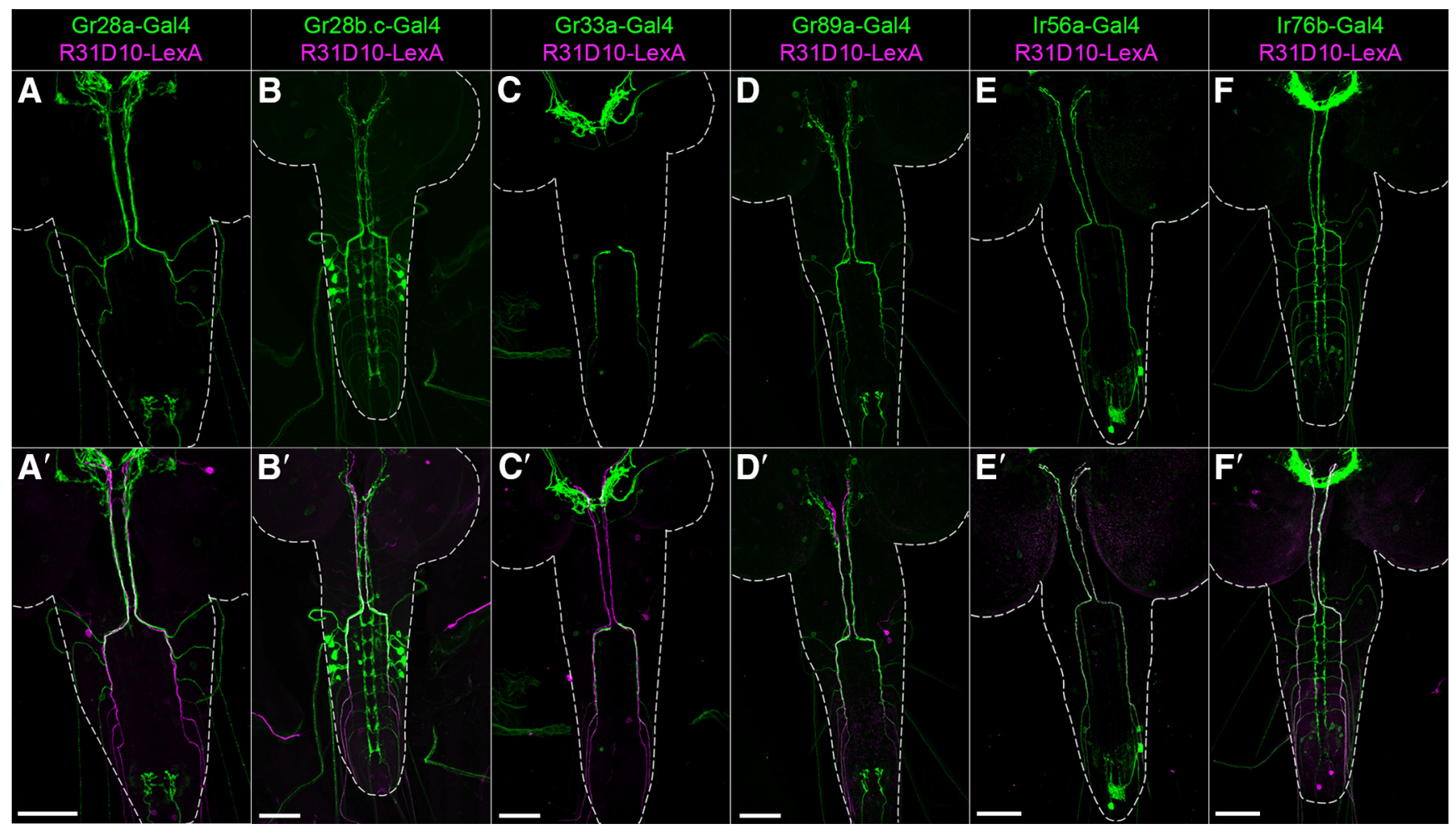

Figure 3. GR and IR GAL4 reporters label td neurons. $A-F$, CNS images showing expression of Gr-Gal4 and Ir-Gal4 lines that label td neurons. $\boldsymbol{A}^{\prime}-\boldsymbol{F}^{\prime}$, Same images dual-labeled with $R 31010-L$ LexA, which labels a subset of td neurons. In addition to td neurons, most of the GAL4 lines labeled gustatory neurons from the head $(\boldsymbol{A}-\boldsymbol{D}, \boldsymbol{F})$, and a few lines labeled terminal sensory neurons $(\boldsymbol{A}, \boldsymbol{D})$, $\mathrm{IV}$ sensory neurons $(\boldsymbol{B})$, external sensory neurons $(\boldsymbol{F})$, or CNS neurons $(\boldsymbol{B}, \boldsymbol{E})$. Scale bars, $50 \mu \mathrm{m}$

were expressed outside of larval head taste organs (Kwon et al., 2011). We found that 4 of these Gr-Gal4s are expressed in td neuron subsets (Fig. 3; Table 1). In Drosophila, functional GR complexes require the expression of multiple GR subunits. Almost all GR-expressing td neurons show expression of multiple GR drivers, suggesting GR expression in td neurons could be functionally relevant. We considered the caveat that Gr-Gal4 promoter fusions may not reflect GR expression in td neurons. Arguing against this possibility, a Gr33a $a^{\text {Gal4 }}$ knock-in line (Moon et al., 2009) showed expression in td neurons in the same pattern as the promoter fusion. In addition to GRs, the IR20a clade of ionotropic receptors (IRs) are proposed to be gustatory and pheromone receptors (Bray and Amrein, 2003; Koh et al., 2014; Joseph and Carlson, 2015). A previous analysis of the expression patterns of these IR drivers identified IR56a-Gal4 expression in neurons associated with the trachea (Stewart et al., 2015). We confirmed the expression of IR56a in a specific subset of td neurons (Table 1 ). We also identified another IR driver, Ir76b-Gal4, that is expressed in a subset of td neurons. Overall, these data suggest that chemosensory receptor genes are expressed in td neurons.

\section{td axon projections and GR/IR GAL4 driver expression correlate with cell identity and segment of origin}

The GAL4 lines we identified label cohorts of fasciculated td axons, obscuring single-axon projection patterns. To determine the axonal morphologies of all individual td neurons, we generated Flybow and MCFO clones (Hadjieconomou et al., 2011; Nern et al., 2015) (Fig. 4A-M). We examined multiple clones from each of the $13 \mathrm{v}$ 'td neurons in the larva (two neurons per hemisegment from A1 to A6, and one neuron per hemisegment in A7). Clonal analysis revealed two main subtypes of v'td neurons with different axon projections in the CNS. All axons project along the canonical td tract described above (Fig. 2B) but terminated at different positions along this tract. Among v'td 2 neurons, axon projections were heterogeneous. Neurons in the more anterior abdominal segments A1-A3 projected to the SEZ (Fig. 4A-C), neurons in A4 and A5 projected just short of the dorsomedial fascicle (Fig. $4 D$, $E$; see also Fig. 2C'), and neurons in A6 projected only along the ventrolateral fascicle (Fig. $4 F$ ). Segment A7 contains only one td neuron and its axon terminated along the ventrolateral fascicle, supporting a v'td2 identity (Fig. $4 G$ ). We also noted that the $\mathrm{A} 7 \mathrm{td}$ axon often sprouted collateral branches that projected medially (Fig. 4G). By contrast, all v'td1 neurons extend axons along the td tract to the dorsomedial fascicle and then anteriorly to the SEZ (Fig. 4H-M). At the SEZ, the axons turn slightly laterally, defasciculate, and terminate as a tuft of processes (Fig. 2B). td identity (i.e., v'td1 or v'td2) and axon projections correlated with GR and IR driver expression patterns (Table 1). v'td1 neurons generally expressed IR drivers (Ir56a and Ir76b) but did not express GR drivers, with the exception of the A1 v'td1, which expressed Gr28a-Gal4. v'td2 neurons that project to the SEZ and VNC shared expression of Gr28b.cGal4 and Gr89a-Gal4 but showed divergent expression of Gr28a-Gal4 (in SEZ-projecting v'td2s) and Gr33a-Gal4 (in VNC-projecting v'td2s).

In summary, these data reveal distinctions between v'td 1 and v'td2 axon targets and gene expression patterns. In addition, we find distinctions in the projections of v'td2 depending on segment of origin. Our results reveal diversity of v'td neurons and support the possibility of multiple functions. 


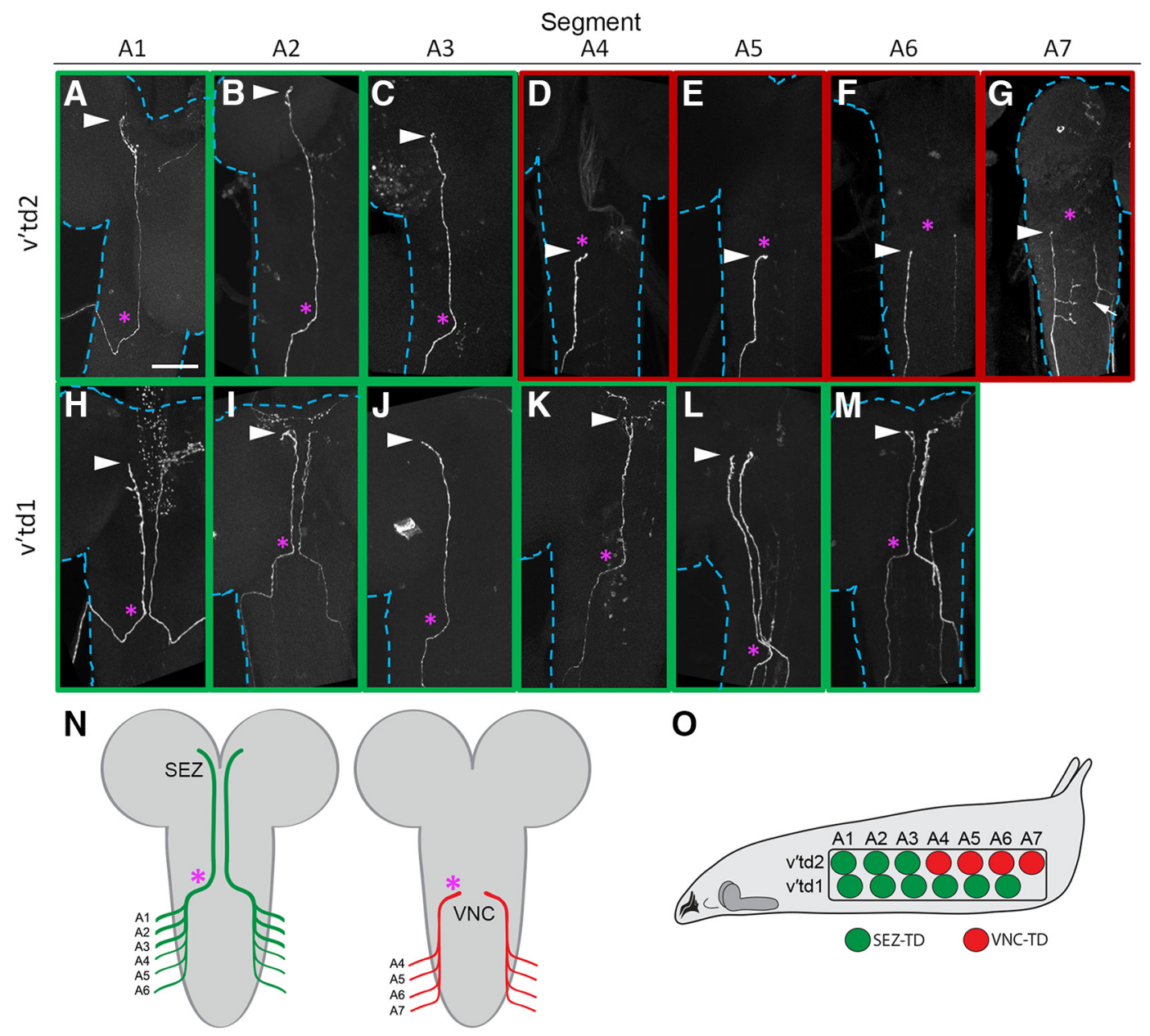

Figure 4. td axon projections correlate with cell identity and segment of origin. $A-M$, Individual central axon projections of all $13 \mathrm{td}$ sensory neurons. Labeled single td neurons were generated using the MCFO technique. Magenta asterisks indicate the approximate location of the T3 neuromere. Arrowhead indicates axon termination. For all image panels, axon of interest is shown on the left side of the CNS. Blue dashed lines outline the CNS. G, White arrow indicates collateral branches from the A7 v'td2. $\boldsymbol{N}, \mathbf{0}$, Schematic summarizing the axon projections ( $\boldsymbol{N}$ ) of SEZ-targeting td neurons (green; SEZ-TDs) and VNC-targeting td neurons (red; VNC-TDs), and the segment of origin and identity of these neurons in the larva body ( $\mathbf{0}$ ). Magenta asterisks indicate the approximate location of the T3 neuromere, as in $\boldsymbol{A}-\boldsymbol{M}$. Scale bars: $\boldsymbol{A}-\boldsymbol{M}, 50 \mu \mathrm{m}$.

\section{OK282-Gal4 is expressed in SEZ targeting td neurons and maps to the $p d m 3$ locus}

Given our identification of distinct morphologies and gene expression patterns among td neurons, we next examined factors that diversify subtypes. As a starting point, we examined enhancer trap GAL4 lines that showed td neuron expression. Lines in our GAL4 collection rarely labeled all td neurons and instead showed cell-specific and segment-specific expression. We examined one line, OK282-Gal4, in more detail. OK282-Gal4 drove expression of $U A S-m C D 8:: G F P$ in v'td 2 in $\mathrm{A} 1$, both td neurons in A2 segments, and v'td1 in segments A3-A6 (Fig. 5A,E). Other than these td neurons, the only other body sensory neuron labeled was a nearby external sensory neuron. Thus, most, but not all, SEZ-projecting td neurons showed OK282-Gal4 expression, but no VNC-projecting td neurons showed expression. We performed splinkerette PCR (Potter and Luo, 2010) to identify the location of the GAL4 insertion. Molecular mapping placed the insertion $27 \mathrm{bp}$ upstream of the first exon of the $p d m 3$ gene (Fig. $5 B) . P d m 3$ is a POU-homeodomain transcription factor that is important for sensory axon targeting and olfactory receptor gene expression in the olfactory system, and targeting of ring neuron axons in the ellipsoid body (Tichy et al., 2008; Chen et al., 2012). The expression pattern of OK282-Gal4 was highly specific and appeared to faithfully report the expression pattern of Pdm3 in both the PNS and CNS based on anti-Pdm3 labeling of OK282Gal4, UAS-mCD8::GFP larvae (Fig. 5C,D). Importantly, antiPdm3 labeled each td neuron marked by OK282-Gal4-driven reporter expression (Fig. 5C). We therefore refer to OK282-Gal4 as pdm $3^{\mathrm{OK} 282}$-Gal4. Based on expression and known roles in axon targeting, we considered $\mathrm{Pdm} 3$ as a strong candidate regulator of td neuron morphogenesis.

\section{$p d m 3$ regulates terminal targeting of td neurons}

The expression pattern of Pdm 3 within td neurons correlates well with axon targeting. Specifically, Pdm3 is expressed in most SEZtargeting td neurons, but not in VNC-targeting td neurons (Fig. $5 E)$. We performed gain- and loss-of-function experiments to test the sufficiency and necessity of $\mathrm{Pdm} 3$ in td axon targeting. First, we asked whether ectopic Pdm3 expression in VNCtargeting td neurons alters axon targeting. R22C07-Gal4 labels $v^{\prime} t d 2$ neurons in A4-A6, which are Pdm3-negative and target the VNC (Fig. 6B). Misexpression of Pdm3 under the control of R22C07-Gal4 promoted ectopic extension of bundled td axons anteriorly beyond the VNC and into the SEZ (Fig. 6C). The maximum distance of extension of the axons was not significantly different from $\mathrm{Pdm}^{+}$SEZ-targeting v'td1 neurons in A4-A6 


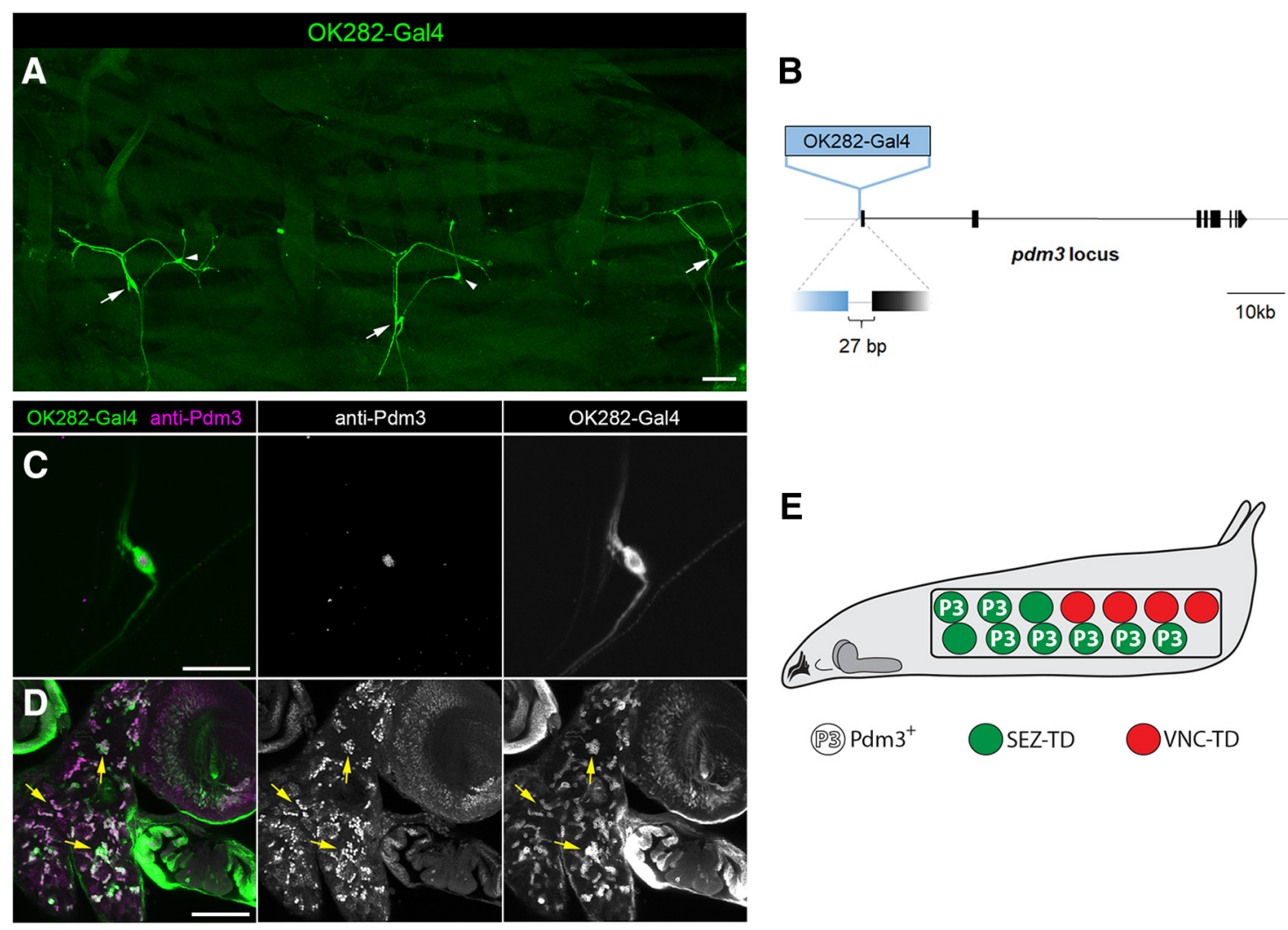

Figure 5. $P d m 3$ is expressed in SEZ-targeting td neurons. A, Expression of OK282-Gal4 $>$ UAS-mCD8::GFP in td neurons (arrow) and a nearby external sensory neuron in the periphery (arrowhead). Image spans three abdominal hemisegments. B, OK282-Gal4 is inserted upstream of the pdm3 locus. C, D, OK282-Gal4 expression pattern matches anti-Pdm3 labeling in td neurons (C) and in CNS (D). Yellow arrows indicate some areas of the CNS with high overlap between the two expression patterns. $\boldsymbol{E}$, Schematic summarizing expression pattern of Pdm3 in td neurons, as determined by pdm ${ }^{\text {OK2282 }}$-Gal4 expression pattern and anti-Pdm3 labeling. Pdm3 (P3) is expressed in most of the SEZ-targeting td neurons (green circles), but not in the VNC-targeting td neurons (red circles). Scale bars: $A, D, 50 \mu \mathrm{m} ; C, 20 \mu \mathrm{m}$.

(labeled by R31D10-LexA; Fig. 6A,D). We observed thinning at the anterior parts of the retargeted axon bundle, suggesting that some, but not all, overextended td axons project all the way to the SEZ. These data suggest that Pdm3 expression is sufficient to switch td axon targeting from the VNC toward the SEZ. In addition, we wondered whether this switch is accompanied by changes in synaptic connectivity. Because VNC axons do not normally make synapses with targets in the SEZ, coexpression of Brp.short and UAS-pdm3 provided a simple assay for altered synapse distribution. We observed Brp signal in the SEZ and in the thoracic neuromeres of the nerve cord in overextended axons, suggesting that VNC neurons that have been retargeted are competent to form synapses in anterior regions of the VNC and in the SEZ (Fig. 6E). Thus, Pdm3 is sufficient for formation of longrange axon projections in td neurons, and for establishing sensory connectivity in the upper VNC and SEZ.

To determine whether Pdm 3 expression was sufficient to promote SEZ projections in non-td axons, we drove expression in nociceptive Class IV (cIV) dendritic arborization neurons, where Pdm3 is not normally expressed, using $p p k$-Gal4. cIV sensory axons normally project directly to ventromedial neuropil and remain largely within their segment of origin (Grueber et al., 2007; Gerhard et al., 2017). Misexpression of Pdm3 did not disrupt expression of the $p p k$-Gal4 reporter. However, we observed extensive axon-targeting defects in cIV neurons, most prominently defasciculation, axon wandering away from the ventral neuropil, and thinning of the axon scaffold near the midline (Fig. $6 F, G)$. cIV axon terminals still targeted the medial neuropil and did not make an initial "td-like" anterior turn at the lateral part of the neuropil, suggesting that Pdm3 does not on its own determine initial td-like axon trajectories (Fig. 6F, G). This conclusion is also consistent with expression of $\mathrm{Pdm} 3$ in only a subset of $\mathrm{td}$ neurons, although all td neurons take the same initial trajectory. Some stray cIV axons projected anteriorly beyond the T1 neuropil and toward the SEZ (Fig. 6F, G). Thus, Pdm3 promotes projection toward the SEZ most robustly in td neurons, suggesting context dependence of axon guidance activity rather than a strict sufficiency of Pdm3 for SEZ projection.

We next examined the necessity of Pdm 3 for td axon targeting. We used R31D10-LexA to label three $\mathrm{Pdm} 3^{+}$td neurons that project to the SEZ (Fig. $7 A$ ). In $p d m 3^{f 00828}$ mutants, td axons took apparently normal projections along the lateral tract, made a normal dorsomedial turn at T3 and projected anteriorly (Fig. $7 B$ ), but axon terminals collapsed around the midline (Fig. $7 B$ ). This result suggests that $\mathrm{Pdm} 3$ is not necessary for projection to the SEZ but is required for correct axon targeting within the SEZ.

In addition to expression in td neurons, Pdm3 is also expressed in CNS glial cells and neurons and, consistent with prior results (Chen et al., 2012), the VNC showed an elongation phenotype in $p d m 3^{f 00828}$ mutants. To reduce Pdm3 function more selectively in neurons, we expressed UAS-pdm3RNAi using a pan-neuronal elav-Gal4 driver, and used R31D10-LexA to label SEZ td axons. We reasoned that elav-Gal4 would allow early enough expression in postmitotic neurons to assess roles in axon guidance or targeting. Consistent with this, we confirmed knockdown of Pdm3 expression in larval td neurons (Fig. 7E). In addition, neuronal knockdown using elav-Gal4 did not lead to the elongated VNC phenotype (Fig. $7 F$ ). In elav-Gal4>pdm3RNAi 

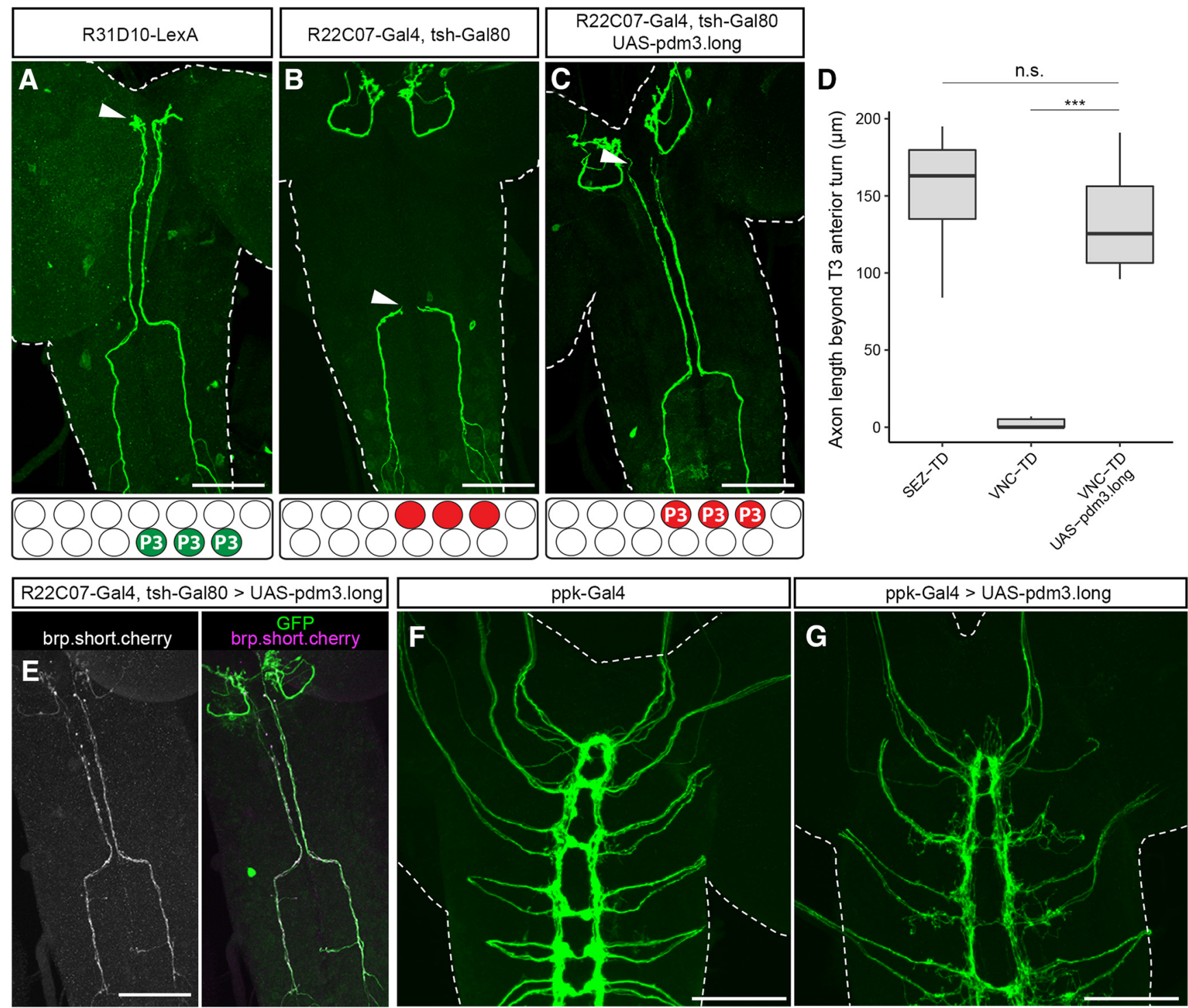

Figure 6. Misexpression of Pdm 3 in VNC-targeting td neurons promotes SEZ targeting. $A-C$, td axons in control $(\boldsymbol{A}, \boldsymbol{B})$ and upon ectopic Pdm 3 expression $(\boldsymbol{C})$ conditions. Arrowhead indicates termination of td axons. Below each confocal image are keys showing SEZ-targeting td neurons labeled by R31D10-LexA (A; green) or VNC-targeting td neurons labeled by R22C07-Gal4 (B; red). P3, Pdm3 expression. Ectopic Pdm3 expression using R22C07-Gal4 switches td axon targeting from the VNC to the SEZ (C). D, Quantification of the length of the axon bundle beyond the axons' anterior turn at T3. Kruskal-Wallis test showed a significant difference of means $\left(H_{(2)}=24.40, p<0.0001\right)$. Post hoc pairwise comparisons showed that VNC-targeting td neurons ectopically expressing $P d m 3$ have increased axon lengths $\left({ }^{* * *} p<0.0001\right.$, Mann-Whitney $U$ test, $\left.U<0.0001\right)$, which did not differ significantly from the length of SEZ-targeting td neurons $(p=0.332$, Mann-Whitney $U$ test, $U=48$ ). $n=12$ animals per condition. $\boldsymbol{E}$, Ectopic expression of Pdm 3 in $R 22$ C07-Gal4 neurons promotes presynaptic structures in the overextended axon segments. $F, G$, Ectopic expression of Pdm3 in non-td sensory neurons ( $\mathrm{IV}$ nociceptive neurons) causes defasciculation and axon wandering. Boxplots represent median (middle line) and 25th to 75th percentile, with whiskers extending to the most extreme data point within 1.5 times the interquartile range of the hinge. Scale bars, $50 \mu \mathrm{m}$.

animals, td axons in the SEZ collapsed around the midline (Fig. 7C). Thus, in both $p d m 3$ mutants and pan-neuronal RNAi knockdown animals, td axon terminals exhibited mistargeting, as seen by incorrect contact of the midline by axons (Fig. 7D). We did not observe defects in the morphogenesis of td dendrites in pdm $3^{f 00828}$-null mutants (Fig. 7G), suggesting that $\mathrm{Pdm} 3$ is dispensable for td dendritogenesis. Overall, our results suggest that $\mathrm{Pdm} 3$ expression in $\mathrm{td}$ neurons is sufficient to promote SEZ axon targeting but might normally function in a redundant fashion. By contrast, $\mathrm{Pdm} 3$ appears to be essential for fine targeting of $\mathrm{td}$ axons in the SEZ.

\section{Ectopic Pdm3 expression suppresses Gr33a-QF reporter expression}

Pdm3 could be regulating axon projection identity or neuronal fate in general. We therefore asked whether Pdm3 regulates broader aspects of td neuronal identity. Given that SEZprojecting and VNC-projecting td neurons differentially express some GRs and IRs, we manipulated Pdm3 expression and asked whether chemosensory receptor expression in td neurons was altered. We focused on the expression of Ir76b, Gr28a (both expressed within SEZ-td population), and Gr33a (expressed within VNC-td population). We did not observe a change in the expression of Ir76b-Gal4, Gr28a-Gal4, or Gr33a $a^{\text {Gal4 }}$ reporter lines in $p d m 3$ mutants, indicating that $\mathrm{Pdm} 3$ is not necessary for GR/IR reporter expression (Fig. 8A). We next used R22C07-Gal4 to drive ectopic Pdm3 expression in VNC-projecting A4-A6 v'td2 neurons, and used QF reporters (Zhang et al., 2013; Choi et al., 2016) to assess GR/IR expression. In control background, Ir76b-QF expression matched Ir76b-Gal4 expression; Gr28a-QF2 

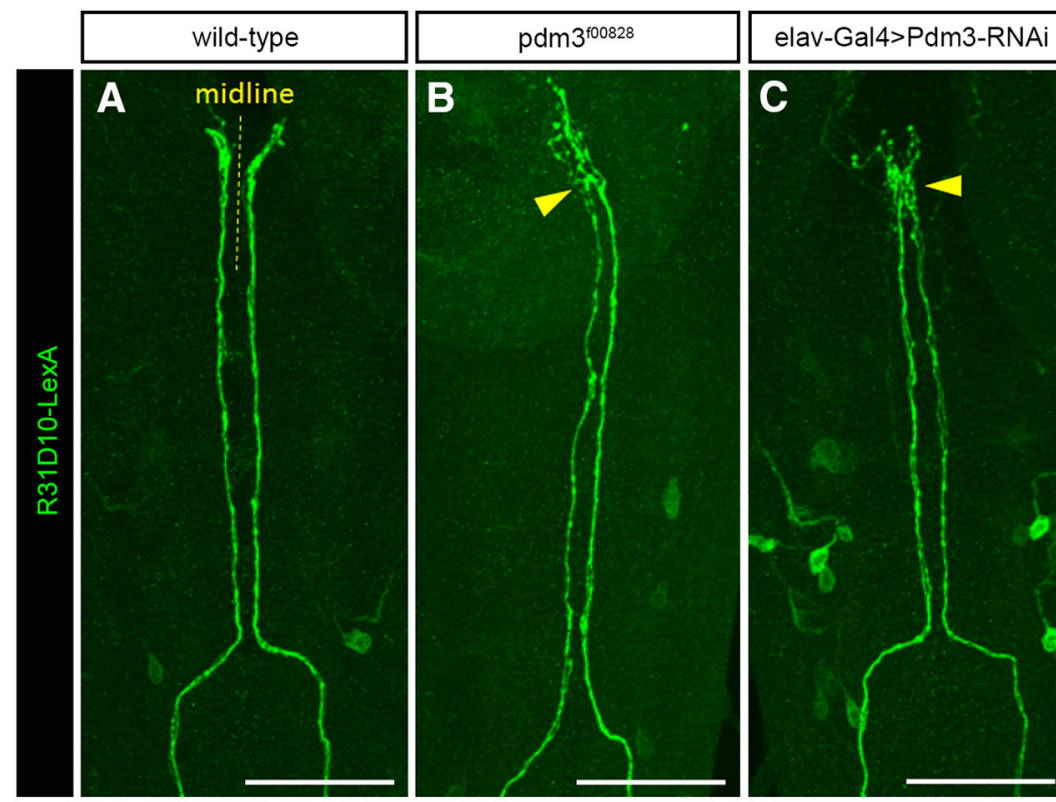

\section{$\mathbf{E}$}

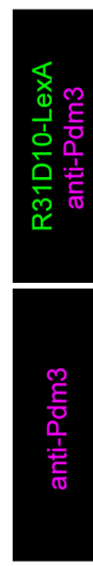

wild-type

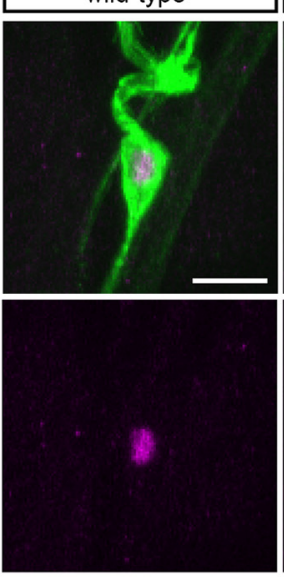

elav-Gal4>Pdm3-RNAi

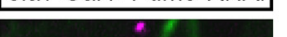

\begin{abstract}
$\mathbf{F}$
\end{abstract}

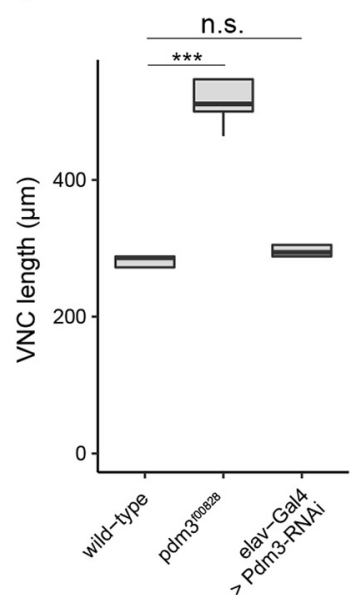

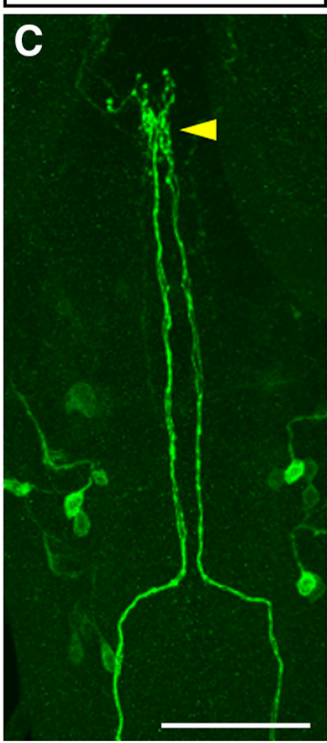

D

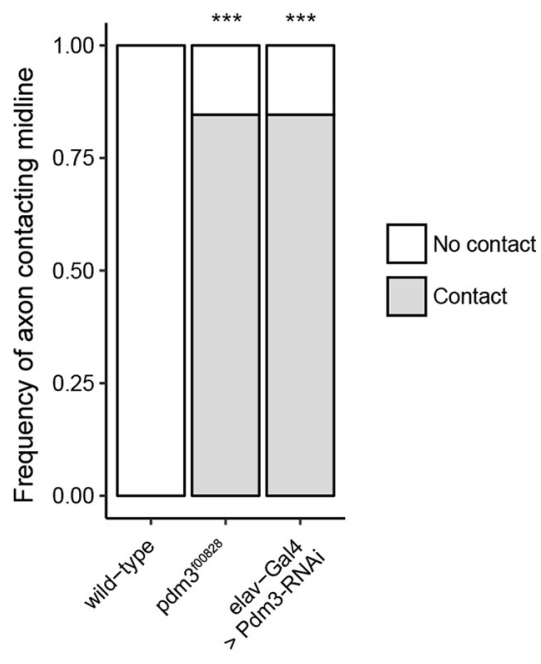

G
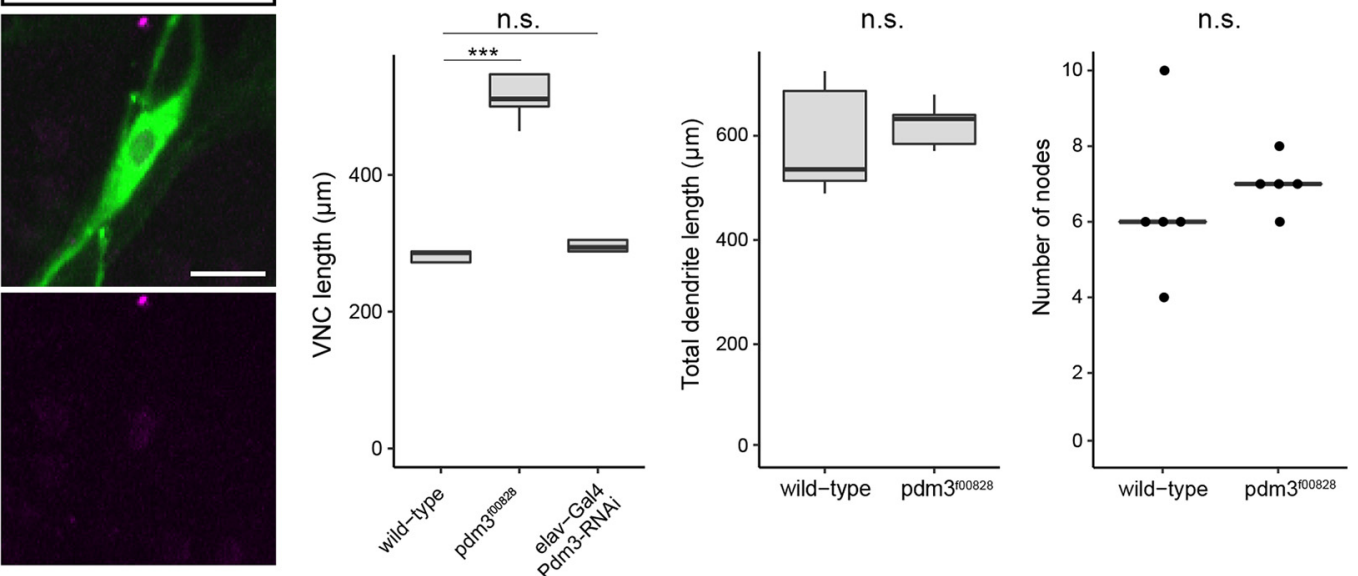

Figure 7. Pdm 3 is required for correct axon targeting within the SEZ. $A-C$, $t d$ axons in control and Pdm 3 loss-of-function conditions. In control condition (A), td neurons labeled by R31D10-LexA project axons to the SEZ, where the axons turn slightly laterally away from the midline. In $p d m 3$ mutant $(\boldsymbol{B})$ and pan-neuronal Pdm3 RNAi knockdown conditions ( $\boldsymbol{C}$, td axon terminals extend toward the SEZ but incorrectly contact the midline (yellow arrowheads). $D$, Quantification of midline contacts by td axon terminals. Increased axon midline contacts were observed in $p d m 3$ mutant $\left(\chi^{2}(1, N=26)=19.07,{ }^{* * *} p<0.0001\right)$ and pan-neuronal Pdm3 RNAi knockdown $\left(\chi^{2}(1, N=26)=19.07,{ }^{* * *} p<0.0001\right)$ conditions. $n=13$ animals per condition. $E$, Confirmation of Pdm3 knockdown in td neurons. $F$, The VNC is abnormally elongated in $p d m 3$ mutant larvae, but not upon pan-neuronal knockdown of Pdm3. Kruskal-Wallis test showed significant difference of means $\left(H_{(2)}=9.89, p=0.007\right)$. Post hoc pairwise comparisons showed increased VNC lengths in $p d m 3$ mutants $\left({ }^{* * *} p<0.0001\right.$, Mann-Whitney $U$ test, $\left.U<0.0001\right)$, but not pan-neuronal Pdm3 RNAi knockdown animals $(p=0.590$, Mann-Whitney $U$ test, $U=7.5) . n=5$ animals per condition. $G$, Loss of Pdm3 does not lead to morphological defects in td dendrites as measured by total dendrite lengths $(p=0.602$, Mann-Whitney $U$ test, $U=10)$ and node counts $(p=0.190$, Mann-Whitney $U$ test, $U=6.5)$. Analysis of A5 v'td1. $n=$ 5 animals per condition. Boxplots represent median (middle line) and 25th to 75 th percentile, with whiskers extending to the most extreme data point within 1.5 times the interquartile range of the hinge. Scale bars: $A-C, 50 \mu \mathrm{m} ; \boldsymbol{E}, 10 \mu \mathrm{m}$.

and Gr33a-QF expression largely matched their respective GAL4 reporter expression, although expression differed slightly (Fig. $8 A, B)$. We found that ectopic Pdm 3 expression in VNC-td neurons using R22C07-Gal4>UAS-pdm3 decreased the frequency with which Gr33a-QF reporter labeled A4-A6 v'td2 neurons from 100\% to $31 \%$ (Fig. $8 B, C$ ). Ectopic Pdm3 expression did not affect Ir76b-QF or Gr28a-QF2 expression (Fig. 8B). Thus, Pdm3 expression is sufficient to suppress VNC-td GR reporter expression, although other inductive factors may be necessary to induce SEZ-td GR/IR expression. Together, these results suggest that $\mathrm{Pdm} 3$ regulates both axon targeting and GR expression aspects of td neuronal identity.

\section{Discussion}

td neuron anatomy, development, and functions are largely uncharacterized. Here we describe their dendrite and axon organization and identify a transcriptional basis for the development of their long-range axons that target to the SEZ. This system provides a novel opportunity to study the genetic basis of complex long-range sensory axon navigation within a neuropil, and the connectivity and function of interoceptive sensory neurons.

High-resolution studies of sensory axon morphology in embryos identified unusual axon projections of $\mathrm{td}$ neurons beyond their segment of origin to a common target in thoracic neuromeres (Merritt and Whitington, 1995). Whether this neuromere 
A

\begin{tabular}{|c|c|c|c|c|c|c|c|c|c|c|c|c|c|c|}
\hline & & A1 & A2 & $\mathrm{A} 3$ & A4 & A5 & A6 & A1 & A2 & A3 & A4 & A5 & A6 & A7 \\
\hline \multirow{2}{*}{ |r76b-Gal4 } & \multirow{2}{*}{$\begin{array}{l}\text { wt } \\
\text { pdm3 mutant }\end{array}$} & & + & + & + & + & + & & & & & & & \\
\hline & & & + & + & + & + & + & & & & & & & \\
\hline \multirow{2}{*}{ Gr28a-Gal4 } & \multirow{2}{*}{$\begin{array}{l}\text { wt } \\
\text { pdm3 mutant }\end{array}$} & + & & & & & & + & + & + & & & & \\
\hline & & + & & & & & & + & + & + & & & & \\
\hline \multirow{2}{*}{ Gr33a ${ }^{\text {Gal4 }}$} & \multirow{2}{*}{$\begin{array}{l}\text { wt } \\
p d m 3 \text { mutant }\end{array}$} & & & & & & & & & & + & + & + & \\
\hline & & & & & & & & & & & + & + & + & \\
\hline
\end{tabular}

B

\begin{tabular}{|c|c|c|c|c|c|c|c|c|c|c|c|c|c|c|}
\hline & & A1 & $A 2$ & A3 & A4 & A5 & A6 & A1 & A2 & A3 & A4 & A5 & A6 & A7 \\
\hline \multirow{2}{*}{ Ir76b-QF } & \multirow{2}{*}{$\begin{array}{l}\text { wt } \\
\text { Ectopic Pdm3 }\end{array}$} & & + & + & + & + & + & & & & & & & \\
\hline & & & + & + & + & + & + & & & & & & & \\
\hline \multirow{2}{*}{ Gr28a-QF2 } & \multirow{2}{*}{$\begin{array}{l}\text { wt } \\
\text { Ectopic Pdm3 }\end{array}$} & & & & & & & + & + & + & & & & \\
\hline & & & & & & & & + & + & + & & & & \\
\hline \multirow{2}{*}{ Gr33a-QF } & \multirow{2}{*}{$\begin{array}{l}\text { wt } \\
\text { Ectopic Pdm3 }\end{array}$} & & & & & & & & & + & + & + & + & + \\
\hline & & & & & & & & & & + & - & - & - & + \\
\hline
\end{tabular}

C

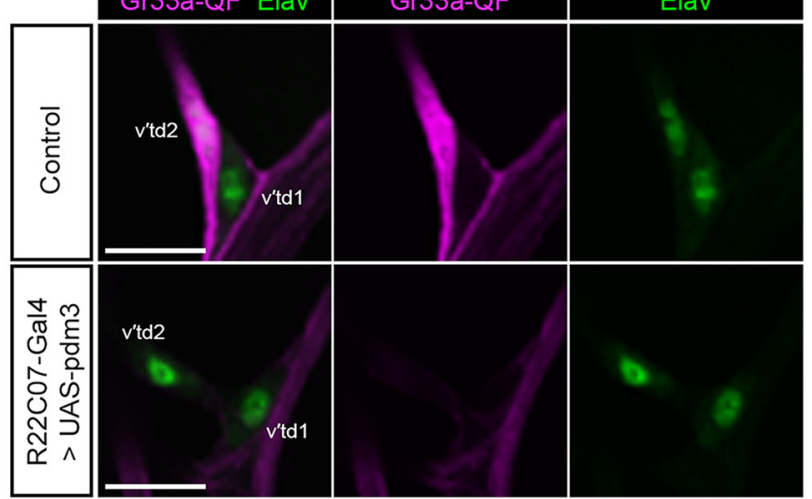

Figure 8. Misexpression of $\mathrm{Pdm} 3$ in VNC-tds suppresses Gr33a-QF reporter expression. $\boldsymbol{A}, \boldsymbol{B}$, Summary of GR/IR reporter expression in wild-type, pdm3 mutant $(\boldsymbol{A})$, and ectopic Pdm3 expression $(\boldsymbol{B})$ conditions. Green and red cells represent SEZ- and VNC-targeting td neurons, respectively. + , Cells with expression (100\% of cell labeled, $n>3$ animals); - , cells with reduced expression ( $n>3$ animals). $\boldsymbol{B}$, Cells with blue outline indicate td neurons misexpressing Pdm3 through R22C07-Gal4> UAS-pdm3. Gr33a-QF labeled v'td2 in A4-A6 with $100 \%$ frequency ( $n=7$ animals, 42 total cells examined) in control condition and only $31 \%$ frequency ( $n=7$ animals, 42 total cells examined) in ectopic Pdm3 condition. C, Expression of Gr33aQF $>$ QUAS-mtdTomato in td neurons in control and ectopic Pdm3 expression conditions. Gr33a-QF also labels glial cells, near td and adjacent nerves. Anti-Elav labels td nuclei. Images show td neurons in A5. Scale bars, $10 \mu \mathrm{m}$.

represented an intermediate or terminal axon target was unknown because mature td axon projections in the third instar larva were not described. Here we show that all td neurons make long-range projections but have dichotomous terminal zones anteriorly in the SEZ and in the VNC. The SEZ receives chemosensory inputs and contains numerous peptidergic fibers (Kwon et al., 2011; Schlegel et al., 2016). Based on their location along trachea, $t d$ neurons were proposed to function as proprioceptors or gas sensors (Bodmer and Jan, 1987; Morton et al., 2008), although the function of td neurons is as yet unknown. Our anatomical data are more consistent with roles for td neurons as internal chemosensors. We note that axons that project to the SEZ form en passant synapses throughout the VNC, suggesting distributed input to central circuits. SEZ- and VNC-targeting axons could conceivably share postsynaptic partners in the VNC, with SEZ-targeting axons connecting with an additional population of targets in the SEZ, although precise connectivity remains to be determined. A recent electron microscopic study of the SEZ identified ascending sensory projections that form synapses with a subset of peptidergic Hugin neurons (Schlegel et al., 2016).
These sensory projections likely correspond to a subset of td neurons. Functional interrogation of this Hugin circuit and reconstruction of additional downstream targets (Schneider-Mizell et al., 2016) of SEZ- and VNC-projecting td neurons will provide insights into possible roles for the td system in behavior and physiology.

We identified expression of multiple GR and IR reporters in td neurons. This finding, together with our anatomical data, suggests that td neurons may function to sense internal chemical stimuli. In Drosophila, the combinatorial coexpression of specific GRs determines the tuning of gustatory neurons to specific ligands (Lee et al., 2009; Shim et al., 2015; Sung et al., 2017). The patterns of coexpressed GRs that we observed in td neurons have not been observed in other gustatory neurons (Kwon et al., 2011; Choi et al., 2016), suggesting possible tuning to novel ligands. Two GRs that appear to be expressed in td neurons, Gr33a and Gr89a, are expressed in all adult bitter neurons (Weiss et al., 2011), and Gr33a is broadly required for responses to aversive cues in the context of feeding (Moon et al., 2009). These GRs have been proposed to function as "core bitter coreceptors" (Moon et al., 2009). It is possible that at least a subset of $t d$ neurons may detect aversive chemical stimuli. Given that td dendrites appear to be bathed in hemolymph and associated with the trachea, $\mathrm{td}$ neurons may detect both dissolved circulating stimuli (e.g., ingested toxins, metabolites, electrolytes) and gaseous stimuli (e.g., $\mathrm{CO}_{2}, \mathrm{O}_{2}$ ). The expression of a reporter for Ir76b, a detector of low salt (Zhang et al., 2013), and oxygen-sensitive guanylyl cyclase (Langlais et al., 2004; Morton et al., 2008) in different subsets of td neurons is consistent with this idea. We speculate that td neurons may detect chemical imbalances and relay signals to the SEZ and VNC to elicit behavioral or physiological responses to restore homeostasis. Neurons in the SEZ could regulate feeding (Hückesfeld et al., 2016), and neurons in the VNC could regulate locomotion (Schoofs et al., 2014; Kohsaka et al., 2017) or fluid balance (Santos et al., 2007; Cognigni et al., 2011). In mammals, lunginnervating sensory neurons comprise molecularly distinct subtypes with different anatomical projections and functions (Chang et al., 2015). Here, we show that larval Drosophila tracheainnervating sensory neurons similarly comprise molecularly distinct subtypes with distinct axon projections. Future studies to image and manipulate td activity, and disrupt chemosensory receptor gene function, should clarify the sensory functions of $t d$ neurons and the underlying molecular mechanisms.

We uncover multiple levels of specificity of td neuron dendrite-substrate relationships, including strict association with a tracheal substrate, arborization across specific tracheal branches, and dendritic specializations at tracheal fusion cells. The factors that specify sensory dendrite organization of td neurons are unknown and do not appear to include Pdm3. Whether dendrite specializations are important for detection of chemicals in the tracheal lumen or whether trachea merely serve as an attachment site to allow sensing of abdominal hemolymph status is not clear (Bodmer and Jan, 1987; Merritt and Whitington, 1995). The positioning of $\mathrm{td}$ dendrites may place them out of direct contact with the tracheal interior; however, association across tracheal cells could still permit sensing of tracheal physiology. Future studies to monitor tracheal system and td dendrite development will help to sort out mechanisms of dendrite-substrate interactions and the importance of this association for td neuron function.

Many of the guidance decisions made by sensory axons involve decisions to terminate at specific mediolateral and dorsoventral positions or in specific neuropil layers (Clandinin and Zipursky, 2002; Zlatic et al., 2003, 2009). For td axons, the guid- 
ance decisions are complex. Single td axons switch between medial and lateral positions, and dorsal and ventral positions and do so at specific locations along their length. Moreover, the terminal position of td axons varies according to cell identity and segment of origin. We predict that studies of td neurons may be especially useful for understanding sequential and regionally restricted guidance switches in axons, a model more akin to long-range projections, such as vertebrate corticospinal tract axons that navigate multiple choice points (Finger et al., 2002), than other locally projecting Drosophila sensory axons.

We provide initial insight into one major choice of td axons: the choice to project, or not, to far anterior regions of the CNS (SEZ). We find that the Pdm3 transcription factor is expressed in most, but not all, td neurons that project to the SEZ and is expressed in none of the td neurons that terminate in the VNC. Ectopic Pdm3 expression promoted anterior axon growth along the canonical td axon path, indicating that $\mathrm{Pdm} 3$ expression is sufficient for SEZ projections. This effect depends on sensory context because misexpression of Pdm3 in cIV dendritic arborization neurons did not convert axons to a td-like projection, but rather led to axon defasciculation, overgrowth, and axon straying, occasionally into the SEZ. Loss of Pdm3 led to modest disruptions of terminal targeting in SEZ-projecting tds, suggesting sufficiency, but redundancy with other factors, in SEZ targeting. We noted specific patterns of axon-axon segregation among axons that project to the SEZ and those that project to the VNC. Thus, in addition to the possibility that Pdm3 functions as a growth-promoting factor, other explanations could account for $\mathrm{Pdm} 3$ misexpression phenotypes, such as promoting specific patterns of axon-axon interactions that underlie pathfinding to anterior CNS.

Our results extend the roles for Pdm3 in axon targeting and chemosensory receptor expression. Prior studies identified roles for Pdm3 in targeting of olfactory sensory neurons, in olfactory receptor expression (Tichy et al., 2008) and in ellipsoid ring (R) neuron axon targeting (Chen et al., 2012). In R neurons, Pdm3 controls axon terminal targeting, without impacting dendritic arborization, cell fate determination, or initial axon outgrowth. Our results for td neurons support a role in axon terminal growth and targeting, or maintenance, and in regulation of GR expression. Thus, we demonstrate that $\mathrm{Pdm} 3$ regulates multiple aspects of td cellular identity, consistent with prior findings in the olfactory system (Tichy et al., 2008). With respect to fine terminal targeting, one potential role for Pdm3 may be to inhibit midline contact of sensory axon terminals, which could account for the Pdm 3 loss-of-function phenotype in td neurons and part of the Pdm3 misexpression phenotype in cIV neurons. The normal functions of Pdm3 in different cell types suggest context-dependent roles to promote terminal targeting. Identifying whether conserved transcriptional targets are shared between these different systems will be an important future step. Studies of Pdm3 might reveal how axon initial growth, pathfinding, terminal targeting, and maintenance are regulated in a modular fashion across different neurons, which could be important not only for axon wiring during development but also for regeneration.

\section{References}

Bauke AC, Sasse S, Matzat T, Klämbt C (2015) A transcriptional network controlling glial development in the Drosophila visual system. Development 142:2184-2193. CrossRef Medline

Bodmer R, Jan YN (1987) Morphological differentiation of the embryonic peripheral neurons in Drosophila. Roux Arch Dev Biol 196:69-77. CrossRef Medline

Bray S, Amrein H (2003) A putative Drosophila pheromone receptor expressed in male-specific taste neurons is required for efficient courtship. Neuron 39:1019-1029. CrossRef Medline

Chang RB, Strochlic DE, Williams EK, Umans BD, Liberles SD (2015) Vagal sensory neuron subtypes that differentially control breathing. Cell 161: 622-633. CrossRef Medline

Chen CK, Chen WY, Chien CT (2012) The POU-domain protein Pdm3 regulates axonal targeting of $\mathrm{R}$ neurons in the Drosophila ellipsoid body. Dev Neurobiol 72:1422-1432. CrossRef Medline

Choi J, van Giesen L, Choi MS, Kang K, Sprecher SG, Kwon JY (2016) A pair of pharyngeal gustatory receptor neurons regulates caffeine-dependent ingestion in Drosophila larvae. Front Cell Neurosci 10:181. CrossRef Medline

Clandinin TR, Zipursky SL (2002) Making connections in the fly visual system. Neuron 35:827-841. CrossRef Medline

Cognigni P, Bailey AP, Miguel-Aliaga I (2011) Enteric neurons and systemic signals couple nutritional and reproductive status with intestinal homeostasis. Cell Metab 13:92-104. CrossRef Medline

Coll AP, Farooqi IS, O'Rahilly S (2007) The hormonal control of food intake. Cell 129:251-262. CrossRef Medline

Corty MM, Matthews BJ, Grueber WB (2009) Molecules and mechanisms of dendrite development in Drosophila. Development 136:1049-1061. CrossRef Medline

Corty MM, Tam J, Grueber WB (2016) Dendritic diversification through transcription factor-mediated suppression of alternative morphologies. Development 143:1351-1362. CrossRef Medline

Craig AD (2003) Interoception: the sense of the physiological condition of the body. Curr Opin Neurobiol 13:500-505. CrossRef Medline

Critchley HD, Harrison NA (2013) Visceral influences on brain and behavior. Neuron 77:624-638. CrossRef Medline

Erkman L, McEvilly RJ, Luo L, Ryan AK, Hooshmand F, O'Connell SM, Keithley EM, Rapaport DH, Ryan AF, Rosenfeld MG (1996) Role of transcription factors brn-3.1 and brn-3.2 in auditory and visual system development. Nature 381:603-606. CrossRef Medline

Finger JH, Bronson RT, Harris B, Johnson K, Przyborski SA, Ackerman SL (2002) The netrin 1 receptors Unc5h3 and dcc are necessary at multiple choice points for the guidance of corticospinal tract axons. J Neurosci 22:10346-10356. Medline

Gerhard S, Andrade I, Fetter RD, Cardona A, Schneider-Mizell CM (2017) Conserved neural circuit structure across Drosophila larval development revealed by comparative connectomics. Elife 6:e29089. CrossRef Medline

Gohl DM, Silies MA, Gao XJ, Bhalerao S, Luongo FJ, Lin CC, Potter CJ, Clandinin TR (2011) A versatile in vivo system for directed dissection of gene expression patterns. Nat Methods 8:231-237. CrossRef Medline

Grueber WB, Ye B, Yang CH, Younger S, Borden K, Jan LY, Jan YN (2007) Projections of Drosophila multidendritic neurons in the central nervous system: links with peripheral dendrite morphology. Development 134: 55-64. CrossRef Medline

Hadjieconomou D, Rotkopf S, Alexandre C, Bell DM, Dickson BJ, Salecker I (2011) Flybow: genetic multicolor cell labeling for neural circuit analysis in Drosophila melanogaster. Nat Methods 8:260-266. CrossRef Medline

Hückesfeld S, Peters M, Pankratz MJ (2016) Central relay of bitter taste to the protocerebrum by peptidergic interneurons in the Drosophila brain. Nat Commun 7:12796. CrossRef Medline

Jenett A, Rubin GM, Ngo TT, Shepherd D, Murphy C, Dionne H, Pfeiffer BD, Cavallaro A, Hall D, Jeter J, Iyer N, Fetter D, Hausenfluck JH, Peng H, Trautman ET, Svirskas RR, Myers EW, Iwinski ZR, Aso Y, DePasquale GM, et al. (2012) A GAL4-driver line resource for Drosophila neurobiology. Cell Rep 2:991-1001. CrossRef Medline

Joseph RM, Carlson JR (2015) Drosophila chemoreceptors: a molecular interface between the chemical world and the brain. Trends Genet 31:683695. CrossRef Medline

Kim ME, Shrestha BR, Blazeski R, Mason CA, Grueber WB (2012) Integrins establish dendrite-substrate relationships that promote dendritic selfavoidance and patterning in Drosophila sensory neurons. Neuron 73:7991. CrossRef Medline

Koh TW, He Z, Gorur-Shandilya S, Menuz K, Larter NK, Stewart S, Carlson JR (2014) The Drosophila IR20a clade of ionotropic receptors are candidate taste and pheromone receptors. Neuron 83:850-865. CrossRef Medline 
Kohsaka H, Guertin PA, Nose A (2017) Neural circuits underlying fly larval locomotion. Curr Pharm Des 23:1722-1733. CrossRef Medline

Komiyama T, Johnson WA, Luo L, Jefferis GS (2003) From lineage to wiring specificity: POU domain transcription factors control precise connections of Drosophila olfactory projection neurons. Cell 112:157-167. CrossRef Medline

Kwon JY, Dahanukar A, Weiss LA, Carlson JR (2011) Molecular and cellular organization of the taste system in the Drosophila larva. J Neurosci 31: 15300-15309. CrossRef Medline

Kwon JY, Dahanukar A, Weiss LA, Carlson JR (2014) A map of taste neuron projections in the Drosophila CNS. J Biosci 39:565-574. CrossRef Medline

Landgraf M, Sánchez-Soriano N, Technau GM, Urban J, Prokop A (2003) Charting the Drosophila neuropile: a strategy for the standardised characterisation of genetically amenable neurites. Dev Biol 260:207-225. CrossRef Medline

Langlais KK, Stewart JA, Morton DB (2004) Preliminary characterization of two atypical soluble guanylyl cyclases in the central and peripheral nervous system of Drosophila melanogaster. J Exp Biol 207:2323-2338. CrossRef Medline

Lee Y, Moon SJ, Montell C (2009) Multiple gustatory receptors required for the caffeine response in Drosophila. Proc Natl Acad Sci U S A 106:44954500. CrossRef Medline

Lin CC, Potter CJ (2016) Editing transgenic DNA components by inducible gene replacement in Drosophila melanogaster. Genetics 203:1613-1628. CrossRef Medline

Manning G, Krasnow MA (1993) Development of the Drosophila tracheal system. In The Development of Drosophila melanogaster, M Bate, A Martínez-Arias (eds), pp 609-685. Cold Spring Harbor, NY: Cold Spring Harbor Laboratory Press.

Matthews BJ, Kim ME, Flanagan JJ, Hattori D, Clemens JC, Zipursky SL, Grueber WB (2007) Dendrite self-avoidance is controlled by dscam. Cell 129:593-604. CrossRef Medline

McEvilly RJ, Erkman L, Luo L, Sawchenko PE, Ryan AF, Rosenfeld MG (1996) Requirement for brn-3.0 in differentiation and survival of sensory and motor neurons. Nature 384:574-577. CrossRef Medline

Merritt DJ, Whitington PM (1995) Central projections of sensory neurons in the Drosophila embryo correlate with sensory modality, soma position, and proneural gene function. J Neurosci 15:1755-1767. Medline

Minokoshi Y, Alquier T, Furukawa N, Kim YB, Lee A, Xue B, Mu J, Foufelle F, Ferré P, Birnbaum MJ, Stuck BJ, Kahn BB (2004) AMP-kinase regulates food intake by responding to hormonal and nutrient signals in the hypothalamus. Nature 428:569-574. CrossRef Medline

Moon SJ, Lee Y, Jiao Y, Montell C (2009) A Drosophila gustatory receptor essential for aversive taste and inhibiting male-to-male courtship. Curr Biol 19:1623-1627. CrossRef Medline

Morton DB, Stewart JA, Langlais KK, Clemens-Grisham RA, Vermehren A (2008) Synaptic transmission in neurons that express the Drosophila atypical soluble guanylyl cyclases, Gyc-89Da and Gyc-89Db, is necessary for the successful completion of larval and adult ecdysis. J Exp Biol 211: 1645-1656. CrossRef Medline

Murphey RK, Possidente D, Pollack G, Merritt DJ (1989) Modality-specific axonal projections in the CNS of the flies phormia and Drosophila. J Comp Neurol 290:185-200. CrossRef Medline

Nern A, Pfeiffer BD, Rubin GM (2015) Optimized tools for multicolor stochastic labeling reveal diverse stereotyped cell arrangements in the fly visual system. Proc Natl Acad Sci U S A 112:E2967-E2976. CrossRef Medline

Pfluger HJ, Braunig P, Hustert R (1988) The organization of mechanosensory neuropiles in locust thoracic ganglia. Philos Trans R Soc Lond B Biol Sci 321:1. CrossRef

Potter CJ, Luo L (2010) Splinkerette PCR for mapping transposable elements in Drosophila. PLoS One 5:e10168. CrossRef Medline

Santos JG, Vömel M, Struck R, Homberg U, Nässel DR, Wegener C (2007) Neuroarchitecture of peptidergic systems in the larval ventral ganglion of Drosophila melanogaster. PLoS One 2:e695. CrossRef Medline

Schlegel P, Texada MJ, Miroschnikow A, Schoofs A, Hückesfeld S, Peters M, Schneider-Mizell CM, Lacin H, Li F, Fetter RD, Truman JW, Cardona A, Pankratz MJ (2016) Synaptic transmission parallels neuromodulation in a central food-intake circuit. Elife 5:e16799. CrossRef Medline

Schmid A, Hallermann S, Kittel RJ, Khorramshahi O, Frolich AM, Quentin C, Rasse TM, Mertel S, Heckmann M, Sigrist SJ (2008) Activity-dependent site-specific changes of glutamate receptor composition in vivo. Nat Neurosci 11:659-666.

Schneider-Mizell CM, Gerhard S, Longair M, Kazimiers T, Li F, Zwart MF, Champion A, Midgley FM, Fetter RD, Saalfeld S, Cardona A (2016) Quantitative neuroanatomy for connectomics in Drosophila. Elife 5:e12059. CrossRef Medline

Schoofs A, Hückesfeld S, Schlegel P, Miroschnikow A, Peters M, Zeymer M, Spiess R, Chiang AS, Pankratz MJ (2014) Selection of motor programs for suppressing food intake and inducing locomotion in the Drosophila brain. PLoS Biol 12:e1001893. CrossRef Medline

Shim J, Lee Y, Jeong YT, Kim Y, Lee MG, Montell C, Moon SJ (2015) The full repertoire of Drosophila gustatory receptors for detecting an aversive compound. Nat Commun 6:8867. CrossRef Medline

Stewart S, Koh TW, Ghosh AC, Carlson JR (2015) Candidate ionotropic taste receptors in the Drosophila larva. Proc Natl Acad Sci U S A 112: 4195-4201. CrossRef Medline

Sung HY, Jeong YT, Lim JY, Kim H, Oh SM, Hwang SW, Kwon JY, Moon SJ (2017) Heterogeneity in the Drosophila gustatory receptor complexes that detect aversive compounds. Nat Commun 8:1484. CrossRef Medline

Tichy AL, Ray A, Carlson JR (2008) A new Drosophila POU gene, pdm3, acts in odor receptor expression and axon targeting of olfactory neurons. J Neurosci 28:7121-7129. CrossRef Medline

Vosshall LB, Wong AM, Axel R (2000) An olfactory sensory map in the fly brain. Cell 102:147-159. CrossRef Medline

Weiss LA, Dahanukar A, Kwon JY, Banerjee D, Carlson JR (2011) The molecular and cellular basis of bitter taste in Drosophila. Neuron 69:258-272. CrossRef Medline

Yang CH, Rumpf S, Xiang Y, Gordon MD, Song W, Jan LY, Jan YN (2009) Control of the postmating behavioral switch in Drosophila females by internal sensory neurons. Neuron 61:519-526. CrossRef Medline

Zhang YV, Ni J, Montell C (2013) The molecular basis for attractive salttaste coding in Drosophila. Science 340:1334-1338. CrossRef Medline

Zlatic M, Landgraf M, Bate M (2003) Genetic specification of axonal arbors: atonal regulates robo3 to position terminal branches in the Drosophila nervous system. Neuron 37:41-51. CrossRef Medline

Zlatic M, Li F, Strigini M, Grueber W, Bate M (2009) Positional cues in the Drosophila nerve cord: semaphorins pattern the dorso-ventral axis. PLoS Biol 7:e1000135. CrossRef Medline 